\title{
Discovery of Fluorescent Cyanopyridine and Deazalumazine Dyes using Small Molecule Macroarrays
}

Matthew D. Bowman, Megan M. Jacobson, and Helen E. Blackwell*

Department of Chemistry, University of Wisconsin-Madison, 1101 University Avenue, Madison, WI 53706-1322

e-mail: blackwell@chem.wisc.edu

\section{Supporting Information.}

General experimental information .......................................................................... S-2

Solution-phase synthesis of standard compounds (3e, 3f, 3n-p, 3q, 6c, and $\mathbf{6 t})$................ S-4

Derivatization of planar cellulose supports .................................................................. S-9

Synthesis of chalcone macroarray 1 ....................................................................... S-10

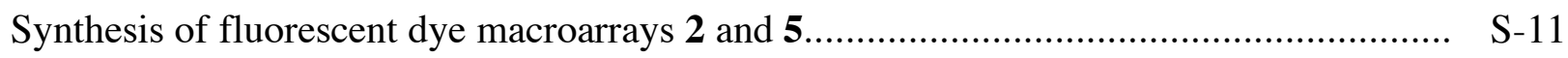

Spectral characterization methods .......................................................................... S-12

Representative spectral characterization data for $\mathbf{3 e}, \mathbf{3 f}$, and $\mathbf{6 c} \ldots \ldots \ldots \ldots \ldots \ldots \ldots \ldots \ldots \ldots \ldots \ldots \ldots . . . . . . . . . . . . . . . . . .13$

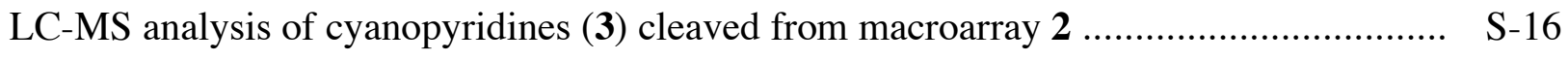

LC-MS analysis of deazalumazines (6) cleaved from macroarray 5 .............................. S-17

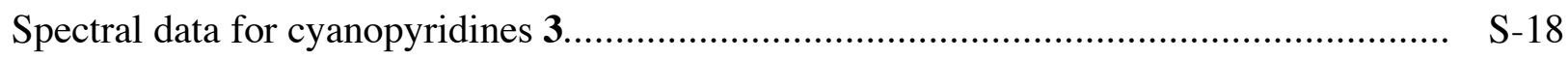

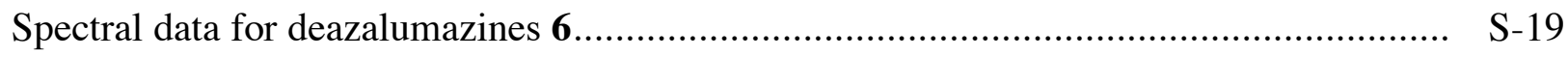

Dependence of the spectral properties of cyanopyridines $\mathbf{3 e}, \mathbf{3 f}, \mathbf{3 n}$, and $\mathbf{3 o}$ on $\mathrm{pH} \ldots \ldots \ldots \ldots . \quad \mathrm{S}-20$

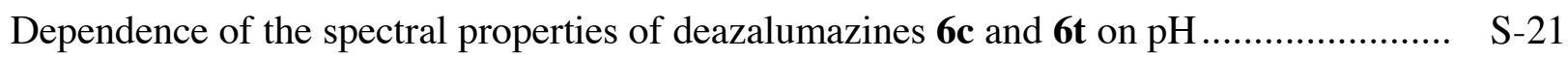

Dependence of the spectral properties of dyes $\mathbf{3 e}, \mathbf{3 f}, \mathbf{3 o}, \mathbf{6 c}$, and $\mathbf{6 t}$ on solvent ................ S-22

References and notes.................................................................................... S-23

\footnotetext{
* To whom correspondence should be addressed.
} 


\section{General experimental information.}

${ }^{1} \mathrm{H}$ NMR and ${ }^{13} \mathrm{C}$ NMR spectra were recorded on a Bruker AC-300 spectrometer in deuterated solvents at $300 \mathrm{MHz}$ and $75 \mathrm{~Hz}$, respectively. Chemical shifts are reported in parts per million (ppm, $\delta)$ using tetramethyl silane (TMS) as a reference $(0.0 \mathrm{ppm})$. Couplings are reported in hertz. LC-MS (ESI) were obtained using a Shimadzu LCMS-2010 (Columbia, MD) equipped with two pumps (LC-10ADvp), controller (SCL-10Avp), autoinjector (SIL-10ADvp), UV diode array detector (SPD-M10Avp), and single quadrupole analyzer (by electrospray ionization, ESI). The LC-MS is interfaced with a PC running the Shimadzu LCMSsolution software package (Version 2.04 Su2-H2). A Supelco (Bellefonte, PA) $15 \mathrm{~cm} \times 2.1 \mathrm{~mm} \mathrm{C}-18$ wide-pore reverse phase column was used for all LC-MS work. Standard reverse phase HPLC conditions were as follows: flow rate $=200 \mu \mathrm{L} / \mathrm{min}$; mobile phase $\mathrm{A}=0.4 \%$ formic acid; mobile phase $\mathrm{B}=0.2 \%$ formic acid in acetonitrile. Attenuated total reflectance (ATR)-IR spectra were recorded with a Bruker Tensor 27 spectrometer, outfitted with a single reflection MIRacle Horizontal ATR by Pike Technologies. A ZnSe crystal with spectral range 20,000 to $650 \mathrm{~cm}^{-1}$ was used. UV spectra were recorded using a Cary $50 \mathrm{Scan}$ UV-Vis spectrometer running Cary WinUV 3.00 software. Fluorescence spectra were recorded on a Hitachi F-4500 Fluorescence spectrophotometer using the following parameters: $5.0 \mathrm{~nm}$ excitation slit width, $5.0 \mathrm{~nm}$ emission slit width, $1200 \mathrm{~nm} / \mathrm{min}$ scan speed, 700 V PMT voltage, and $2.0 \mathrm{sec}$ response time. Quantum yields were calculated using standard methods. ${ }^{1}$ Thin layer chromatography (TLC) was performed on silica gel $60 \mathrm{~F}_{254}$ plates (E-5715-7, Merck). Silica gel 60 (230-400 mesh, EM Science) was used for flash column chromatography. ${ }^{2}$ All reported melting points are uncorrected.

All reagents were purchased from commercial sources (Alfa-Aesar, Aldrich, and Acros) and used without further purification. Solvents were purchased from commercial sources (Aldrich and J.T. Baker) and used as is, with the exception of dichloromethane $\left(\mathrm{CH}_{2} \mathrm{Cl}_{2}\right)$, which was distilled over calcium hydride immediately prior to use. Planar cellulose membranes (Whatman 1Chr chromatography paper, $20 \times 20 \mathrm{~cm}$ squares) were purchased from Fischer Scientific and stored in a dessicator at room temperature until ready for use.

Microwave instrumentation. All microwave-assisted reactions were performed in a Milestone MicroSYNTH Labstation multimodal microwave synthesis reactor equipped with a continuous power source $(1000 \mathrm{~W}$ max). This instrument is interfaced with an Ethos MicroSYNTH Lab Terminal PC running EasyWave reaction monitoring software. Using this reactor system, microwave irradiation can be applied to reactions using either power (wattage) control or temperature control. The microwave reactor is equipped with a fiber-optic temperature sensor that allows direct monitoring of the internal temperature of reaction vessels and an infrared sensor (installed in the side wall of the reactor cavity) that can monitor the surface temperature of reaction vessels inside the cavity. ${ }^{3}$ Solvent depths of $c a .1 \mathrm{~cm}$ in the reaction vessel are required for accurate temperature monitoring using the submerged fiber-optic temperature probe. Solution-phase reactions were performed in the MicroSYNTH reactor using specialized $70 \mathrm{~mL}$ Teflon/PEEK reaction vessels. These vessels have appropriate holes in their lids to accommodate the fiber-optic temperature sensor in a protective ceramic sheath.

Planar cellulose supports were irradiated in a shallow Pyrex dish as depicted in the graphic on page S-3 on a rotating turntable inside the MicroSYNTH microwave reactor. Planar supports 
were irradiated routinely using power control, as insufficient solvent volumes were used for accurate temperature monitoring using the fiber-optic probe.

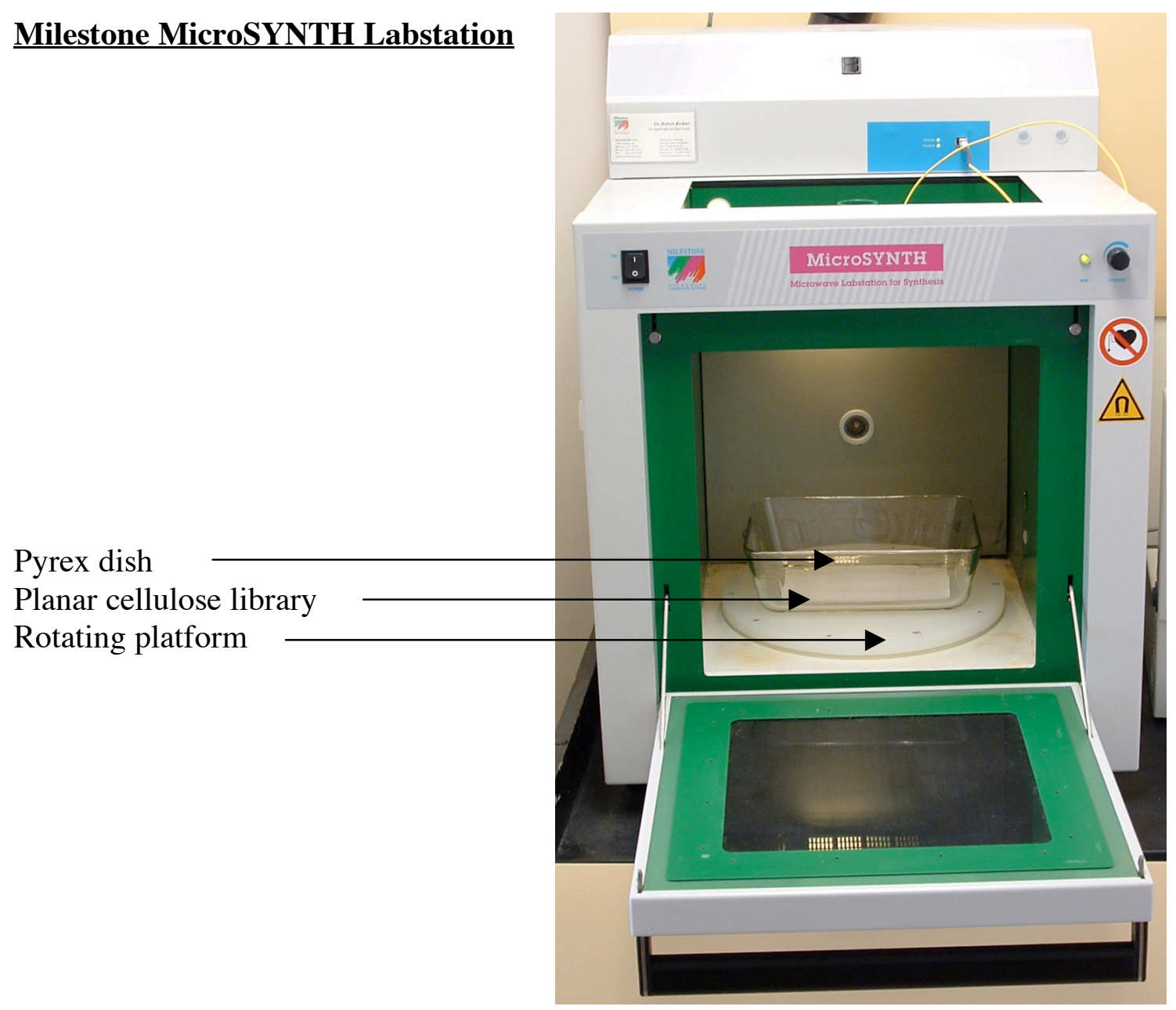

Solid-phase synthesis techniques. Planar cellulose membranes were mixed with reagents at room temperature and washed with solvents in glass vessels on a Lab-Line orbital shaker housed in a fume hood. All spotting procedures used in SPOT-synthesis ${ }^{4}$ were performed manually using Brinkman Eppendorf pipettmen (calibrated for variable solvent delivery (1-10 $\mu \mathrm{L})$ ) and disposable polypropylene pipette tips. 
Solution-phase synthesis of standard compounds (3e, 3f, 3n-p, 3q, 6c, and 6t).

Compound codes C1-C10 are used for compounds not mentioned directly in the text.

1-(4-(tetrahydro-2H-pyran-2-yloxy)phenyl)ethanone (C1). 4-hydroxyacetophenone ( $8.5 \mathrm{~g}$, $62.5 \mathrm{mmol}), 3,4$-dihydropyran $(8.5 \mathrm{~mL}, 100 \mathrm{mmol})$, and $p$-toluenesulfonic acid $(0.5 \mathrm{~g} 5.0 \mathrm{mmol})$ were dissolved in $125 \mathrm{~mL}$ of dichloromethane equipped with a stirbar and stirred for $17 \mathrm{~h}$ at room temperature. The reaction mixture was washed with saturated aq. $\mathrm{Na}_{2} \mathrm{CO}_{3}$, the organic layer was dried over $\mathrm{MgSO}_{4}$, and concentrated under reduced pressure to afford $12.86 \mathrm{~g}$ of pale brown solid. $93 \%$ yield. Characterization matched that reported by Ma and Venanzi. ${ }^{5}$

4-dimethylamino-4'-(tetrahydro-2H-pyran-2-yloxy)chalcone (C2). Compound C1 (2.64 g, $12.0 \mathrm{mmol})$, 4-dimethylaminobenzaldehyde $(1.80 \mathrm{~g}, 12.0 \mathrm{mmol})$ were dissolved in EtOH (40 $\mathrm{mL}$ ) in a $100 \mathrm{~mL}$ round bottom flask. A $2 \mathrm{~mL}$ aliquot of $50 \%(\mathrm{w} / \mathrm{v})$ aqueous $\mathrm{NaOH}$ was added, and the solution was stirred overnight (approximately $18 \mathrm{~h}$ ) at room temperature. The yellow precipitate was filtered and recrystallized from $\mathrm{MeOH}$ to afford $3.3 \mathrm{~g}$ of yellow-orange solid. $78 \%$ yield. TLC: $\mathrm{R}_{\mathrm{f}}=0.31$ (20\% ethyl acetate in hexane); Melting point: $110-111{ }^{\circ} \mathrm{C} ;{ }^{1} \mathrm{H}-\mathrm{NMR}$ (300 MHz, DMSO-d6) $\delta 8.09,7.13\left(\mathrm{AA}^{\prime} \mathrm{XX} X^{\prime}, \mathrm{J}_{\mathrm{AA}}=\mathrm{J}_{\mathrm{XX}}=2.4, \mathrm{~J}_{\mathrm{AX}}=8.7, \mathrm{~J}_{\mathrm{AX}}=0.2 \mathrm{~Hz}, 4 \mathrm{H}\right), 7.63(\mathrm{~m}$, $2 \mathrm{H}), 7.61(\mathrm{~d}, \mathrm{~J}=1.3 \mathrm{~Hz}), 6.72(\mathrm{~m}, 2 \mathrm{H}), 5.6(\mathrm{~m}, 1 \mathrm{H}), 3.73(\mathrm{~m}, 1 \mathrm{H}), 3.59(\mathrm{~m}, 1 \mathrm{H}), 3.02(\mathrm{~s}, 6 \mathrm{H})$, $1.80(\mathrm{~m}, 3 \mathrm{H}), 1.57$ (m, 3H); ${ }^{13} \mathrm{C}-\mathrm{NMR}$ (75 MHz, DMSO-d6) $\delta 187.8,160.7,152.5,145.0,142.5$, 131.2, 130.9, 122.8, 116.8, 116.6, 112.4, 96.22, 62.3, 30.3, 25.3, 19.1; IR (ATR): 2951, 1645, 1599, 1573, 1439, 1400, 1371, 1342, 1287, 1216, 1187, 1161, $1119 \mathrm{~cm}^{-1}$; ESI-MS: expected, 351.2; observed, $\mathrm{m} / \mathrm{z} 352.3\left[\mathrm{M}+\mathrm{H}^{+}\right]$.

4'-hydroxy-4-methoxychalcone (C3). 4'-hydroxyacetophenone (1.63 g, $12 \mathrm{mmol})$ and $p$ anisaldehyde $(1.45 \mathrm{~mL}, 12 \mathrm{mmol})$ were dissolved in $\mathrm{MeOH}(30 \mathrm{~mL})$ in a $70 \mathrm{~mL}$ Teflon/PEEK Milestone microwave reaction vessel. A $2 \mathrm{~mL}$ aliquot of $50 \%$ (w/v) aqueous $\mathrm{NaOH}$ was added, and the solution was stirred until all reactants had dissolved. The reaction vessel was tightly closed and heated (with stirring) using a Milestone microwave reactor from room temperature to $150{ }^{\circ} \mathrm{C}$ over $15 \mathrm{~min}$, held at $150{ }^{\circ} \mathrm{C}$ for $20 \mathrm{~min}$, and allowed to cool to room temperature. The reaction mixture was poured over approximately $30 \mathrm{~g}$ of ice and acidified to $\mathrm{pH} 1.0$ with $1 \mathrm{M}$ $\mathrm{HCl}$, forming a yellow precipitate. The solid was isolated and recrystallized from $\mathrm{MeOH}$ to afford $0.90 \mathrm{~g}$ of golden crystals, $30 \%$ yield. TLC: $\mathrm{R}_{\mathrm{f}}=0.25$ (40\% ethyl acetate in hexane); Melting point: $185-188{ }^{\circ} \mathrm{C}$; ${ }^{1} \mathrm{H}-\mathrm{NMR}(300 \mathrm{MHz}$, DMSO-d6) $\delta 10.35$ (brs, $1 \mathrm{H}$ ), 8.05, 6.89 $\left(\mathrm{AA}^{\prime} \mathrm{XX}, \mathrm{J}_{\mathrm{AA}}=\mathrm{J}_{\mathrm{XX}}=2.4, \mathrm{~J}_{\mathrm{AX}}=8.6, \mathrm{~J}_{\mathrm{AX}}=0.2 \mathrm{~Hz}, 4 \mathrm{H}\right), 7.92,7.00\left(\mathrm{AA}^{\prime} \mathrm{XX}, \mathrm{J}_{\mathrm{AA}}=\mathrm{J}_{\mathrm{XX}}=2.4, \mathrm{~J}_{\mathrm{AX}}=8.6\right.$, $\left.\mathrm{J}_{\mathrm{AX}}=0.2 \mathrm{~Hz}, 4 \mathrm{H}\right), 7.80,7.60(\mathrm{AB}$ peak, $\mathrm{J}=15.5 \mathrm{~Hz}, 2 \mathrm{H}) ;{ }^{13} \mathrm{C}-\mathrm{NMR}(75 \mathrm{MHz}$, DMSO-d6) $\delta 187.7$, 162.7, 161.8, 143.3, 131.7, 131.2m 130.0, 128.207, 120.3, 116.0, 115.0, 56.0; IR (ATR): 3200, 2990, 1643, 1602, 1562, 1512, 1430, 1352, 1286, 1223, 1165, $1046 \mathrm{~cm}^{-1}$; ESI-MS: expected, 254.1; observed, $\mathrm{m} / \mathrm{z} 254.8\left[\mathrm{M}+\mathrm{H}^{+}\right]$.

4-fluoro-4'-hydroxychalcone (C4). 4'-hydroxyacetophenone (1.63 g, $12 \mathrm{mmol})$ and 4fluorobenzaldehyde $(1.28 \mathrm{~mL}, 12 \mathrm{mmol})$ were dissolved in $40 \mathrm{~mL}$ EtOH in a $100 \mathrm{~mL}$ round bottom flask. A $2 \mathrm{~mL}$ aliquot of $50 \%(\mathrm{w} / \mathrm{v})$ aqueous $\mathrm{NaOH}$ was added, and the solution was stirred overnight (approximately $15.5 \mathrm{~h}$ ) at room temperature. Approximately $30 \mathrm{~g}$ of ice were added to the solution, which was acidified to $\mathrm{pH} 4$ with $1 \mathrm{M} \mathrm{HCl}$. The resulting pale yellow precipitate was isolated by filtration and recrystallized from a mixture of EtOH and water to afford $1.53 \mathrm{~g}$ of golden crystals. $54 \%$ yield. TLC: $\mathrm{R}_{\mathrm{f}}=0.30$ (20\% ethyl acetate in hexane); 
Melting point: $177-180{ }^{\circ} \mathrm{C} ;{ }^{1} \mathrm{H}-\mathrm{NMR}(300 \mathrm{MHz}, \mathrm{DMSO}-\mathrm{d6}) \delta 10.39$ (brs, $1 \mathrm{H}$ ) 8.06, 6.90 $\left(\mathrm{AA}^{\prime} \mathrm{XX} \mathrm{X}^{\prime}, \mathrm{J}_{\mathrm{AA}}=\mathrm{J}_{\mathrm{XX}}=2.4, \mathrm{~J}_{\mathrm{AX}}=8.7, \mathrm{~J}_{\mathrm{AX}}=0.3 \mathrm{~Hz}, 4 \mathrm{H}\right), 7.93(\mathrm{~m}, 2 \mathrm{H}), 7.95,7.58$ (AB peak, J= 15.6 $\mathrm{Hz}, 2 \mathrm{H}), 7.28(\mathrm{~m}, 2 \mathrm{H}) ;{ }^{13} \mathrm{C}-\mathrm{NMR}(75 \mathrm{MHz}$, DMSO-d6) $\delta 187.7,163.9$ (d, J=249.4 Hz) 162.9, 142.1, 132.2 (d, J= 2.8 Hz) 131.8, 131.6, (d, J = 9 Hz), 129.8, 122.7, 116.5 (d, J= $22 \mathrm{~Hz}$ ), 116.0; IR (ATR): 3145, 1647, 1568, 1509, 1441, 1417, 1342, 1286, 1219, 1166, $1157 \mathrm{~cm}^{-1}$; ESI-MS: expected, 242.1; observed, $\mathrm{m} / \mathrm{z} 243.1\left[\mathrm{M}+\mathrm{H}^{+}\right]$.

4,4'-dimethoxychalcone (C5). In a $100 \mathrm{~mL}$ round bottom flask, 4-methoxyacetophenone (1.5 g, $10.0 \mathrm{mmol}$ ), p-anisaldehyde (1.63 g, $12.0 \mathrm{mmol}), 2.0 \mathrm{~mL}$ of $50 \%$ aq. $\mathrm{NaOH}$, and $30 \mathrm{~mL}$ of $\mathrm{MeOH}$ were combined. The solution was closed with a stopper and stirred at room temperature for $8 \mathrm{~h}$. The precipitate was filtered, washed with $2.0 \mathrm{~mL}$ of $50 \% \mathrm{aq} . \mathrm{MeOH}$, and allowed to air dry. This procedure generated $2.29 \mathrm{~g}$ of $\mathbf{C 5}$ as a white solid. $85 \%$ yield. TLC: $\mathrm{R}_{\mathrm{f}}=0.50$ (20\% ethyl acetate in hexane); Melting point: 89-90 ${ }^{\circ} \mathrm{C} ;{ }^{1} \mathrm{H}-\mathrm{NMR}(300 \mathrm{MHz}$, DMSOd6) $\delta 8.16,7.08\left(\mathrm{AA}^{\prime} \mathrm{XX}\right.$ ' peak, $\mathrm{J}_{\mathrm{AA}}=\mathrm{J}_{\mathrm{XX}}=2.4, \mathrm{~J}_{\mathrm{AX}}=8.7, \mathrm{~J}_{\mathrm{AX}}=0.2 \mathrm{~Hz}, 4 \mathrm{H}$ ), 7.88, 7.63 (AB peak, $\mathrm{J}=15.3 \mathrm{~Hz}, 2 \mathrm{H}), 7.85,7.02\left(\mathrm{AA}^{\prime} X X^{\prime}\right.$ peak, $\left.\mathrm{J}_{\mathrm{AA}}=\mathrm{J}_{\mathrm{XX}}=2.7, \mathrm{~J}_{\mathrm{AX}}=8.7, \mathrm{~J}_{\mathrm{AX}}=0.2 \mathrm{~Hz}, 4 \mathrm{H}\right), 3.86(\mathrm{~s}, 3 \mathrm{H})$, 3.82 (s, 3H); ${ }^{13} \mathrm{C}-\mathrm{NMR}$ (75 MHz, DMSO-d6) $\delta 187.9,163.7,161.9,143.8,131.4,131.3,128.1$, 120.2, 115.1, 114.6, 56.2, 56.0; IR (ATR): 3550, 3491, 1653, 1593, 1568, 1509, 1463, 1441, 1423, 1335, 1294, 1248, 1215, 1180, 1165, cm ${ }^{-1}$; ESI-MS: expected, 268.1; observed, m/z 269.0 $\left[\mathrm{M}+\mathrm{H}^{+}\right]$.

2-fluoro-4'-methoxychalcone (C6). Synthesized according to the above method for C5: $2.07 \mathrm{~g} ; 65 \%$ yield; TLC: $\mathrm{R}_{\mathrm{f}}=0.56$ (20\% ethyl acetate in hexane); Melting point: $92-93{ }^{\circ} \mathrm{C}$; ${ }^{1} \mathrm{H}$ NMR (300MHz, DMSO-d6) $\delta 8.17,7.10\left(\mathrm{AA}^{\prime} \mathrm{XX}\right.$ ' peak, $\mathrm{J}_{\mathrm{AA}}=\mathrm{J}_{\mathrm{XX}}=2.4, \mathrm{~J}_{\mathrm{AX}}=8.7, \mathrm{~J}_{\mathrm{AX}}=0.3 \mathrm{~Hz}$, $4 \mathrm{H}), 8.13(\mathrm{~m}, 1 \mathrm{H}), 7.51(\mathrm{~m}, 1 \mathrm{H}), 7.32(\mathrm{~m}, 2 \mathrm{H}), 8.09,7.72$ (AB peak, J=15.7 Hz, 2H), 7.85, 7.02 (AA'XX' peak, $\left.\mathrm{J}_{\mathrm{AA}}=\mathrm{J}_{\mathrm{XX}}=2.7, \mathrm{~J}_{\mathrm{AX}}=8.7, \mathrm{~J}_{\mathrm{AX}}=0.2 \mathrm{~Hz}, 4 \mathrm{H}\right), 3.86(\mathrm{~s}, 3 \mathrm{H}), 3.82(\mathrm{~s}, 3 \mathrm{H}) ;{ }^{13} \mathrm{C}-\mathrm{NMR}$ (75 MHz, DMSO-d6) $\delta$ 187.9, 164.1, 161.6 (d, J=255 Hz), 134.9 (d, J=4.5 Hz), 133.1 (d, J=8.0 Hz), 131.6 (d, J=7.4 Hz), 130.9, 129.7, 125.6, 124.9, 123.0 (d, J=14.6 Hz), 116.7 (d, J=23.3 Hz), 114.8, 56.2; IR (ATR): 2977, 1656, 1609, 1573, 1484, 1458, 1423, 1337, 1318, 1285, 1257 , 1230, 1189, $1094 \mathrm{~cm}^{-1}$; ESI-MS: expected, 256.1; observed, m/z $256.9\left[\mathrm{M}+\mathrm{H}^{+}\right]$.

4-fluoro-4'-methoxychalcone (C7). Synthesized according to the above method for C5: $2.68 \mathrm{~g}$; $87 \%$ yield; TLC: $\mathrm{R}_{\mathrm{f}}=0.45$ (40\% ethyl acetate in hexane); Melting point: $107-108{ }^{\circ} \mathrm{C} ;{ }^{1} \mathrm{H}-$ NMR (300 MHz, DMSO-d6) $\delta 8.13,7.05$ (AA'XX' peak, $\mathrm{J}_{\mathrm{AA}}=\mathrm{J}_{\mathrm{XX}}=2.5, \mathrm{~J}_{\mathrm{AX}}=8.7, \mathrm{~J}_{\mathrm{AX}}=0.3 \mathrm{~Hz}$, 4H), 7.93 (m, 2H), 7.87, 7.68 (AB peak, J=15.8 Hz, 2H), 7.26 (m, 2H), 3.81 (s, 3H); ${ }^{13} \mathrm{C}-\mathrm{NMR}$ (75 MHz, DMSO-d6) $\delta$ 188.0, 164 (d, J=248 Hz), 163.9, 142.5, 132.2, 131.7 (d, J=8.6 Hz), 131.6, 131.1, 122.6, 116.5 (d, $22.3 \mathrm{~Hz}), 114.7,56.2$; IR (ATR): 3322, 1655, 1598, 1572, 1508, 1424, 1339, 1315, 1286, 1218, 1189, $1158 \mathrm{~cm}^{-1}$; ESI-MS: expected, 256.1; observed, m/z 256.9 $\left[\mathrm{M}+\mathrm{H}^{+}\right]$.

3',4',4-trimethoxychalcone (C8). Synthesized according to the above method for C5: 1.91 g; 64\% yield; TLC: $\mathrm{R}_{\mathrm{f}}=0.17$ (20\% ethyl acetate in hexane); Melting point: $73-74{ }^{\circ} \mathrm{C} ;{ }^{1} \mathrm{H}-\mathrm{NMR}$ $(300 \mathrm{MHz}, \mathrm{DMSO}-\mathrm{d} 6) \delta 7.88(\mathrm{dd}, \mathrm{J}=8.5,2.0 \mathrm{~Hz}, 1 \mathrm{H}), 7.84,7.01\left(\mathrm{AA}^{\prime} \mathrm{XX}^{\prime}\right.$ peak, $\mathrm{J}_{\mathrm{AA}}=\mathrm{J}_{\mathrm{XX}}=2.2$, $\left.\mathrm{J}_{\mathrm{AX}}=8.9, \mathrm{~J}_{\mathrm{AX}}=-0.1 \mathrm{~Hz}, 4 \mathrm{H}\right), 7.80,7.69(\mathrm{AB}$ peak, $\mathrm{J}=15.5 \mathrm{~Hz}, 2 \mathrm{H}), 7.60(\mathrm{~d}, \mathrm{~J}=2.0 \mathrm{~Hz}, 1 \mathrm{H}), 7.08(\mathrm{~d}$, $\mathrm{J}=8.5 \mathrm{~Hz}, 1 \mathrm{H}), 3.87$ (s, 3H), $3.86(\mathrm{~s}, 3 \mathrm{H}), 3.82(\mathrm{~s}, 3 \mathrm{H}) ;{ }^{13} \mathrm{C}-\mathrm{NMR}(75 \mathrm{MHz}, \mathrm{DMSO}-\mathrm{d} 6) \delta 187.9$, $161.9,153.7,149.5,143.7,131.4,131.3,128.2,123.8,120.1,115.0,111.5,111.4,56.4,56.3$, 56.0; IR (ATR): 3383, 3200, 1649, 1594, 1568, 1513, 1459, 1424, 1336, 1295, 1265, 1248, 1201, 1180, 1163, 1150, 1055, 1027; ESI-MS: expected, 256.1; observed, m/z $256.9\left[\mathrm{M}+\mathrm{H}^{+}\right]$. 
3',4-dimethoxychalcone (C9). Synthesized according to the above method for C5: $1.54 \mathrm{~g}$; $87 \%$ yield; TLC $\mathrm{R}_{\mathrm{f}}=0.28$ (20\% ethyl acetate in hexane); Melting point: $45-47{ }^{\circ} \mathrm{C} ;{ }^{1} \mathrm{H}-\mathrm{NMR}(300$ MHz, DMSO-d6) $\delta 7.98$ (d, J=15.7 Hz), 7.90 (dd, J=8.5, $1.8 \mathrm{~Hz}, 1 \mathrm{H}), 7.84$ (d, J=8.4 Hz, 2H), $7.65(\mathrm{~d}, \mathrm{~J}=15.7 \mathrm{~Hz}, 1 \mathrm{H}), 7.63(\mathrm{~d}, \mathrm{~J}=8.5 \mathrm{~Hz}, 1 \mathrm{H}), 7.58$ (d, J=1.8 Hz, 1H), 7.08 (d, J=8.4 Hz, 2H), 3.85 (s, 3H), 3.83 (s, 3H); ${ }^{13} \mathrm{C}-\mathrm{NMR}$ (75 MHz, DMSO-d6) $\delta 187.8,154.0,149.5,142.4,134.8$, 132.5, 131.4, 131.0, 124.4, 124.2, 123.4, 111.5, 111.3, 56.5, 56.2; IR (ATR): 2998, 2935, 2835, $1655,1584,1566,1513,1488,1462,1455,1425,1339,1320,1295,1281,1250,1216 \mathrm{~cm}^{-1}$; ESIMS: expected, 268.1; observed, $\mathrm{m} / \mathrm{z} 268.9\left[\mathrm{M}+\mathrm{H}^{+}\right]$.

3-cyano-4,6-di(4-methoxyphenyl)-2-methylpyridine (3o). In a $250 \mathrm{~mL}$ round bottom flask equipped with a reflux condenser, 4,4'-dimethoxychalcone (C5, $1.07 \mathrm{~g}, 4.0 \mathrm{mmol})$, 3aminocrotononitrile (328 mg, $4.0 \mathrm{mmol}), \mathrm{NaOH}(160 \mathrm{mg}, 4.0 \mathrm{mmol}$ ), and $160 \mathrm{~mL}$ of EtOH were combined. The solution was refluxed for $8 \mathrm{~h}$. The condenser was removed, and the solution was stirred at room temperature under an oxygen atmosphere. After $20 \mathrm{~min}$, a precipitate formed. After $4 \mathrm{~h}$, the precipitate was filtered, washed with $4.0 \mathrm{~mL}$ of $50 \%$ aq. $\mathrm{MeOH}$, and allowed to air dry. This procedure generated $321 \mathrm{mg}$ of 30 as a white solid. $24 \%$ yield. TLC: $\mathrm{R}_{\mathrm{f}}=0.56(20 \%$ ethyl acetate in hexane); Melting point: 153-154 ${ }^{\circ} \mathrm{C}$; ${ }^{1} \mathrm{H}-\mathrm{NMR}$ (300 MHz, DMSO-d6) $\delta ~ 8.19$,

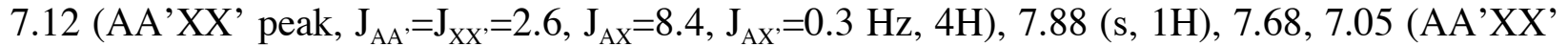
peak, $\left.\mathrm{J}_{\mathrm{AA}},=\mathrm{J}_{\mathrm{XX}}=2.6, \mathrm{~J}_{\mathrm{AX}}=8.6, \mathrm{~J}_{\mathrm{AX}}=0.2 \mathrm{~Hz}, 4 \mathrm{H}\right), 3.83(\mathrm{~s}, 3 \mathrm{H}), 3.82(\mathrm{~s}, 3 \mathrm{H}), 2.76(\mathrm{~s}, 3 \mathrm{H}) ;{ }^{13} \mathrm{C}-\mathrm{NMR}$ (75 MHz, DMSO-d6) $\delta$ 209.0, 162.6, 162.0, 161.4, 160.0, 158.4, 154.2, 130.9, 129.9, 129.1, 118.3, 116.9, 114.7, 56.0, 55.8, 24.5; IR (ATR): 2990, 2901, 2217, 1610, 1575, 1445, 1385, 1345, 1305, 1261, 1169, 1113, 1090, $1058 \mathrm{~cm}^{-1}$; ESI-MS: expected, 330.1; observed, m/z 331.1 $\left[\mathrm{M}+\mathrm{H}^{+}\right]$.

3-cyano-4-(2-fluorophenyl)-6-(4-methoxyphenyl)-2-methylpyridine (3n). Synthesized according to the above method for 3o: $328 \mathrm{mg}$; $26 \%$ yield; TLC: $\mathrm{R}_{\mathrm{f}}=0.60$ (20\% ethyl acetate in hexane); Melting point: $152-153{ }^{\circ} \mathrm{C}$; ${ }^{1} \mathrm{H}-\mathrm{NMR}$ (300 MHz, DMSO-d6) $\delta$ 8.20, 7.05 (AA'XX' peak, $\left.\mathrm{J}_{\mathrm{AA}}=\mathrm{J}_{\mathrm{XX}}=2.6, \mathrm{~J}_{\mathrm{AX}}=8.7, \mathrm{~J}_{\mathrm{AX}}=0.3 \mathrm{~Hz}, 4 \mathrm{H}\right), 7.98(\mathrm{~s}, 1 \mathrm{H}), 7.62(\mathrm{~m}, 2 \mathrm{H}), 7.42(\mathrm{~m}, 2 \mathrm{H}), 3.82(\mathrm{~s}$, 3H), $2.78(\mathrm{~s}, 3 \mathrm{H}) ;{ }^{13 \mathrm{C}}-\mathrm{NMR}\left(75 \mathrm{MHz}, \mathrm{CDCl}_{3}\right) \delta 162.5,161.9,159.5(\mathrm{~d}, \mathrm{~J}=249.9 \mathrm{~Hz}), 158.9$, 148.3, 131.9 (d, J=8.2 Hz), 130.9 (d, J=2.3 Hz), 130.3, 129.2, 124.8 (d, J=3.9 Hz), 124.7, 118.1 (d, $2.2 \mathrm{~Hz}), 117.0,116.8,116.5,144.6,106.4,55.6,24.5$; IR (ATR): 3344, 2990, 2901, 2217 , 1610, 1585, 1539, 1488, 1444, 1386, 1309, 1261, 1220, 1171, $1066 \mathrm{~cm}^{-1}$; ESI-MS: expected, 318.1; observed, $\mathrm{m} / \mathrm{z} 319.0\left[\mathrm{M}+\mathrm{H}^{+}\right]$.

3-cyano-4-(3,4-dimethoxyphenyl)-6-(4-methoxyphenyl)-2-methylpyridine (3q). Synthesized according to the above method for 3o: $545 \mathrm{mg} ; 38 \%$ yield; TLC: $\mathrm{R}_{\mathrm{f}}=0.20(20 \%$ ethyl acetate in hexane); Melting point: 187-188 ${ }^{\circ} \mathrm{C} ;{ }^{1} \mathrm{H}-\mathrm{NMR}$ (300 MHz, DMSO-d6) $\delta 7.95$ (s, $1 \mathrm{H}), 7.86(\mathrm{dd}, \mathrm{J}=8.5,2.1 \mathrm{~Hz}, 1 \mathrm{H}), 7.80(\mathrm{~d}, \mathrm{~J}=2.1 \mathrm{~Hz}), 7.70,7.14\left(\mathrm{AA}^{\prime} X^{\prime}\right.$ ' peak, $\mathrm{J}_{\mathrm{AA}}=\mathrm{J}_{\mathrm{XX}}=2.5$, $\left.\mathrm{J}_{\mathrm{AX}}=8.6, \mathrm{~J}_{\mathrm{AX}}=0.2 \mathrm{~Hz}, 4 \mathrm{H}\right), 7.09(\mathrm{~d}, \mathrm{~J}=8.6 \mathrm{~Hz}, 1 \mathrm{H}), 3.86(\mathrm{~s}, 3 \mathrm{H}), 3.85(\mathrm{~s}, 3 \mathrm{H}), 3.84(\mathrm{~s}, 3 \mathrm{H}), 2.75$ (s, 3H); ${ }^{13} \mathrm{C}-\mathrm{NMR}$ (75 MHz, DMSO-d6) $\delta$ 162.4, 161.3, 158.4, 153.5, 151.7, 149.6, 130.9, 129.1, 121.5, 117.5, 115.0, 112.4, 111.2, 56.4, 56.3, 56.1, 24.8; IR (ATR): 2990, 2901, 2218, 1612, 1573, 1517, 1445, 1384, 1303, 1268, 1237, 1170, 1114, $1058 \mathrm{~cm}^{-1}$; ESI-MS: expected, 360.2; observed, m/z $361.1\left[\mathrm{M}+\mathrm{H}^{+}\right]$.

3-cyano-4-(3-methoxyphenyl)-6-(4-methoxyphenyl)-2-methylpyridine (3p). In a $250 \mathrm{~mL}$ round bottom flask equipped with a reflux condenser, 3',4-dimethoxychalcone (C9, $805 \mathrm{mg}, 3.0$ 
mmol), 3-aminocrotononitrile (246 $\mathrm{mg}, 3.0 \mathrm{mmol})$, sodium hydroxide (120 mg, 3.0 -mmol), and $120 \mathrm{~mL}$ of EtOH were combined. The solution was refluxed for $24 \mathrm{~h}$. The condenser was removed, and the solution was stirred at room temperature under an oxygen atmosphere. After 48 $\mathrm{h}$, the precipitate was filtered, washed with $5.0 \mathrm{~mL}$ of EtOH, and allowed to air dry. $321 \mathrm{mg}$ of white solid were isolated. $32 \%$ yield. TLC $\mathrm{R}_{\mathrm{f}}=0.33$ (20\% ethyl acetate in hexane); Melting point: 151-153 ${ }^{\circ} \mathrm{C}$; ${ }^{1} \mathrm{H}-\mathrm{NMR}\left(300 \mathrm{MHz}, \mathrm{CHCl}_{3}\right) \delta 7.64(\mathrm{~m}, 3 \mathrm{H}), 7.61,7.05$ (AA'XX' peak, $\left.\mathrm{J}_{\mathrm{AA}}=\mathrm{J}_{\mathrm{XX}}=2.5, \mathrm{~J}_{\mathrm{AX}}=8.5, \mathrm{~J}_{\mathrm{AX}}=0.3 \mathrm{~Hz}, 4 \mathrm{H}\right), 7.41(\mathrm{t}, \mathrm{J}=8.1 \mathrm{~Hz}, 1 \mathrm{H}), 7.01$ (ddd, J=8.1, 2.7, 0.9 Hz, $1 \mathrm{H}), 3.90(\mathrm{~s}, 3 \mathrm{H}), 3.89(\mathrm{~s}, 3 \mathrm{H}), 2.90(\mathrm{~s}, 3 \mathrm{H}) ;{ }^{13} \mathrm{C}-\mathrm{NMR}\left(75 \mathrm{MHz}, \mathrm{CHCl}_{3}\right) \delta 163.0,131.2,160.3$, 159.1, 153.7, 139.6, 130.2, 130.1, 128.9, 120.0, 118.1, 117.8, 116.2, 114.7, 113.0, 105.8, 55.7, 24.7; IR (ATR): 3031, 2959, 2845, 2221, 1609, 1602, 1582, 1569, 1541, 1514, 1493, 1475, 1445 , 1426, 1383, 1368, 1328, 1310, 1296, 1257, $1235 \mathrm{~cm}^{-1}$; ESI-MS: expected, 330.1; observed, m/z $330.9\left[\mathrm{M}+\mathrm{H}^{+}\right]$.

3-cyano-4-(4-dimethylaminophenyl)-6-(4-(tetrahydro-2H-pyran-2-yloxy)phenyl)-2methylpyridine (C10). In a $100 \mathrm{~mL}$ round bottom flask equipped with a reflux condenser, 4dimethylamino-4'-(tetrahydro-2H-pyran-2-yloxy)chalcone $\quad(\mathbf{C 2}, 703 \mathrm{mg}, 2.0 \mathrm{mmol}), 3-$ aminocrotononitrile $(328 \mathrm{mg}, 4.0 \mathrm{mmol}), \mathrm{NaOH}(80 \mathrm{mg}, 2.0 \mathrm{mmol})$, and $160 \mathrm{~mL}$ of EtOH were combined. The solution was refluxed for $29 \mathrm{~h}$. The condenser was removed, and the solution was stirred at room temperature under an oxygen atmosphere for $12 \mathrm{~h}$. The precipitate was filtered and allowed to air dry. This procedure generated $366 \mathrm{mg}$ of $\mathbf{C 1 0}$ as a white solid. $44 \%$ yield. TLC: $\mathrm{R}_{\mathrm{f}}=0.33$ (20\% ethyl acetate in hexane); Melting point: $148-150{ }^{\circ} \mathrm{C} ;{ }^{1} \mathrm{H}-\mathrm{NMR}(300 \mathrm{MHz}$, $\left.\mathrm{CDCl}_{3}\right) \delta 8.02,7.17\left(\mathrm{AA}^{\prime} \mathrm{XX}\right.$ ' peak, $\left.\mathrm{J}_{\mathrm{AA}}=\mathrm{J}_{\mathrm{XX}}=2.2, \mathrm{~J}_{\mathrm{AX}}=8.6, \mathrm{~J}_{\mathrm{AX}}=0.1 \mathrm{~Hz}, 4 \mathrm{H}\right), 7.60,6.81$ $\left(\mathrm{AA}^{\prime} \mathrm{XX}\right.$ ' peak, $\left.\mathrm{J}_{\mathrm{AA}}=\mathrm{J}_{\mathrm{XX}}=2.3, \mathrm{~J}_{\mathrm{AX}}=8.6, \mathrm{~J}_{\mathrm{AX}}=0.2 \mathrm{~Hz}, 4 \mathrm{H}\right), 7.59(\mathrm{~s}, 1 \mathrm{H}), 5.51(\mathrm{~m}, 1 \mathrm{H}), 3.90(\mathrm{~m}$, $1 \mathrm{H}), 3.63(\mathrm{~m}, 2 \mathrm{H}), 3.05(\mathrm{~s}, 6 \mathrm{H}), 2.85(\mathrm{~s}, 3 \mathrm{H}), 2.09(\mathrm{~m}, 2 \mathrm{H}), 1.90(\mathrm{~m}, 2 \mathrm{H}), 1.67(\mathrm{~m}, 3 \mathrm{H}) ;{ }^{13} \mathrm{C}-$ NMR $\left(75 \mathrm{MHz}, \mathrm{CDCl}_{3}\right) \delta 207.2,162.7,158.9,158.5,153.8,151.5,131.6,129.6,128.9,123.8$, 118.4, 116.8, 116.5, 112.1, 104.2. 96.3, 62.2, 40.3, 30.7, 30.5, 30.4, 30.2, 30.0, 29.7, 25.3, 24.5, 18.8; IR (ATR): 3195, 2924, 2221, 1679, 1582, 1529, 1435, 1370, 1310, 1241, 1199, 1180, 1142, 1054, $1033 \mathrm{~cm}^{-1}$; ESI-MS: expected, 413.2; observed, m/z 414.2 [M+H'].

3-cyano-4-(4-dimethylaminophenyl)-6-(4-hydroxyphenyl)-2-methylpyridine (3f). In a 4 $\mathrm{mL}$ vial, 3-cyano-4-(4-dimethylaminophenyl)-6-(4-(tetrahydro-2H-pyran-2-yloxy)phenyl)-2methyl-pyridine (C10, $150 \mathrm{mg}, 0.36 \mathrm{mmol}$ ) was dissolved in $1.0 \mathrm{~mL}$ of $20 \%$ trifluoroacetic acid in $\mathrm{CH}_{2} \mathrm{Cl}_{2}$ were combined. The vial was closed with a Teflon-lined cap, shaken, and allowed to stand for $5 \mathrm{~min}$. The solution was concentrated to a dark orange oil under a stream of $\mathrm{N}_{2}$. Trituration with $0.5 \mathrm{~mL}$ of diethyl ether produced $100 \mathrm{mg}$ of $\mathbf{3 f}$ as a dark red solid. $83 \%$ yield. TLC: $\mathrm{R}_{\mathrm{f}}=0.22$ (40\% ethyl acetate in hexane); Melting point: $144-145{ }^{\circ} \mathrm{C} ;{ }^{1} \mathrm{H}-\mathrm{NMR}(300 \mathrm{MHz}$, DMSO): $\delta$ 8.07, 6.87 (AA'XX' peak, $\left.\mathrm{J}_{\mathrm{AA}}=\mathrm{J}_{\mathrm{XX}}=2.4, \mathrm{~J}_{\mathrm{AX}}=13.6, \mathrm{~J}_{\mathrm{AX}}=5.1 \mathrm{~Hz}, 4 \mathrm{H}\right), 7.76(\mathrm{~s}, 1 \mathrm{H})$, 7.61, $6.84\left(\mathrm{AA}^{\prime} \mathrm{XX}\right.$ ' peak, $\left.\mathrm{J}_{\mathrm{AA}}=\mathrm{J}_{\mathrm{XX}}=2.3, \mathrm{~J}_{\mathrm{AX}}=8.9, \mathrm{~J}_{\mathrm{AX}}=0.1 \mathrm{~Hz}, 4 \mathrm{H}\right), 3.00(\mathrm{~s}, 6 \mathrm{H}), 2.74(\mathrm{~s}, 3 \mathrm{H})$; ${ }^{13} \mathrm{C}-\mathrm{NMR}\left(75 \mathrm{MHz}, \mathrm{CDCl}_{3}\right): \delta 162.5,160.4,158.3,153.7,151.9,130.2,129.8,128.7,123.7$, 118.8, 116.3, 116.2, 112.6, 103.4, 31.3, 24.7; IR (ATR): 3195, 2973, 2230, 1678, 1596, 1582, 1528, 1436, 1371, 1241, 1197, 1180, 1142, $1033 \mathrm{~cm}^{-1}$; ESI-MS: expected, 329.2; observed, m/z $330.0\left[\mathrm{M}+\mathrm{H}^{+}\right]$.

3-cyano-4-(4-methoxyphenyl)-6-(4-hydroxyphenyl)-2-methylpyridine (3e). 4'-hydroxy-4methoxychalcone $(\mathbf{C 3}, 508 \mathrm{mg}, 2.0 \mathrm{mmol}), 3$-aminocrotononitrile $(328 \mathrm{mg}, 4.0 \mathrm{mmol}), \mathrm{NaOH}$ (80 mg, $2.0 \mathrm{mmol}$ ), and $40 \mathrm{~mL}$ of EtOH were combined in a $70 \mathrm{~mL}$ Teflon/PEEK Milestone microwave reaction vessel. The reaction vessel was tightly closed and heated (with stirring) 
using a Milestone microwave reactor from room temperature to $150{ }^{\circ} \mathrm{C}$ over $15 \mathrm{~min}$, held at 150 ${ }^{\circ} \mathrm{C}$ for $20 \mathrm{~min}$, and allowed to cool to room temperature. The solution was poured into a $100 \mathrm{~mL}$ round bottom flask. A $4.0 \mathrm{~mL}$ aliquot of $1 \mathrm{~N}$ aq. $\mathrm{HCl}$ was added. The solution was stirred at room temperature under an oxygen atmosphere for $12 \mathrm{~h}$. The precipitate was filtered and allowed to air dry. This procedure generated $110 \mathrm{mg}$ of $\mathbf{3 e}$ as a white solid. $9 \%$ yield. TLC: $\mathrm{R}_{\mathrm{f}}=0.14$ (20\% ethyl acetate in hexane); Melting point: $220-221{ }^{\circ} \mathrm{C}$; ${ }^{1} \mathrm{H}-\mathrm{NMR}(300 \mathrm{MHz}$, Acetone-d6) $\delta 8.18,6.99$

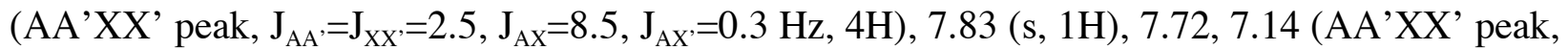
$\left.\mathrm{J}_{\mathrm{AA}}=\mathrm{J}_{\mathrm{XX}}=2.6, \mathrm{~J}_{\mathrm{AX}}=8.5, \mathrm{~J}_{\mathrm{AX}}=0.3 \mathrm{~Hz}, 4 \mathrm{H}\right), 3.85(\mathrm{~s}, 3 \mathrm{H}), 2.75(\mathrm{~s}, 3 \mathrm{H}),{ }^{13} \mathrm{C}-\mathrm{NMR}(75 \mathrm{MHz}$, DMSOd6) $\delta 162.4,161.2,160.6,158.6,153.2,130.8,129.8,129.1,128.5,118.4,116.8,116.3,114.9$, 104.1, 56.1, 24.7; IR (ATR): 3195, 2973, 2221, 1610, 1587, 1533, 1517, 1462, 1382, 1303, 1284, 1252, 1176, 1119, 1048, $1033 \mathrm{~cm}^{-1}$; ESI-MS: expected, 316.1; observed, m/z 316.9 [M+H ${ }^{+}$.

1,3-dimethyl-5-(4-fluorophenyl)-7-(4-hydroxyphenyl)-pyrido[2,3-d]pyrimidine-2,4 (1H, 3H)-dione (6c). 4'-fluoro-4-hydroxychalcone (C4, $480 \mathrm{mg}, 2.0 \mathrm{mmol}$ ), 6-amino-2,3dimethyluracil (4, $310 \mathrm{mg}, 2.0 \mathrm{mmol}), \mathrm{KOH}(112 \mathrm{mg}, 2.0 \mathrm{mmol})$, and $4.0 \mathrm{~mL}$ of EtOH were combined in a $20 \mathrm{~mL}$ vial. The vial was purged with oxygen, sealed with a Teflon-lined cap, and heated at $80{ }^{\circ} \mathrm{C}$ in a conventional drying oven for $16 \mathrm{~h}$. The mixture was allowed to cool to room temperature, after which $2.0 \mathrm{~g}$ of ice was added. After stirring for $15 \mathrm{~min}$, the mixture was filtered and washed with $1.0 \mathrm{~mL}$ of water and $1.0 \mathrm{~mL}$ of EtOH. This procedure generated $113 \mathrm{mg}$ of $\mathbf{6 c}$ as a yellow solid. $15 \%$ yield. TLC: $\mathrm{R}_{\mathrm{f}}=0.28$ (40\% ethyl acetate in hexane); ${ }^{1} \mathrm{H}-\mathrm{NMR}$ (300 MHz, DMSO-d6) $\delta 7.97$ (d, 8.9 Hz, 2H), 7.39 (m, 2H), 7.32 (s, 1H), 7.21 (m, 2H), 6.60 (d, J=8.9 $\mathrm{Hz}, 2 \mathrm{H}), 3.66$ (s, 3H), 3.15 (s, 3H); ${ }^{13} \mathrm{C}-\mathrm{NMR}$ (75 MHz, DMSO-d6) $\delta$ 169.4, 162.5 (d, J=244.4 $\mathrm{Hz}$ ), 160.5, 159.8, 152.7, 152.1, 136.8, 131.0 (d, J=8.2 Hz), 130.1, 122.0, 118.4, 116.3, 114.8 (d, $\mathrm{J}=21.4 \mathrm{~Hz}), 104.0$, 30.3, 28.5; ESI-MS: expected, 377.12; observed, m/z $378.1\left[\mathrm{M}+\mathrm{H}^{+}\right]$.

1,3-dimethyl-5-(4-fluorophenyl)-7-(4-methoxyphenyl)-pyrido[2,3-d]pyrimidine-2,4(1H, 3H)-dione (6t). Synthesized according to the above method for 6c: $460 \mathrm{mg}$. 59\% yield. TLC: $\mathrm{R}_{\mathrm{f}}=0.14$ (20\% ethyl acetate in hexane); Melting point: $240-243{ }^{\circ} \mathrm{C} ;{ }^{1} \mathrm{H}-\mathrm{NMR}\left(300 \mathrm{MHz}, \mathrm{CDCl}_{3}\right)$ d 8.0, 7.01 (AA'XX' peak, $\left.\mathrm{J}_{\mathrm{AA}}=\mathrm{J}_{\mathrm{XX}}=2.5, \mathrm{~J}_{\mathrm{AX}}=8.7, \mathrm{~J}_{\mathrm{AX}}=0.2 \mathrm{~Hz}, 4 \mathrm{H}\right), 7.39(\mathrm{~s}, 1 \mathrm{H}), 7.32(\mathrm{~m}, 2 \mathrm{H})$, 7.15 (m, 2H), 3.89 (s, 3H), 3.87 (s, 3H), 3.39 (s, 3H); ${ }^{13} \mathrm{C}-\mathrm{NMR}\left(75 \mathrm{MHz}, \mathrm{CDCl}_{3}\right) \delta$ 163.2, 162.7, (d, J=246 Hz), 162.0, 160.6, 159.0, 153.9, 151.7 (d, J=22.4 Hz), 135.5 (d, J=2.9 Hz), 129.7 (d, $\mathrm{J}=8.3 \mathrm{~Hz}$ ), 129.1, 117.6, 115.0, 114.8, 114.4, 105.8, 55.5, 30.1, 28.4; IR (ATR): 2989, 2896, 1702, 1660, 1600, 1457, 1439, 1422, 1367, 1287, 1263, 1221, 1165, $1086 \mathrm{~cm}^{-1}$; ESI-MS: expected, 377.12; observed, $\mathrm{m} / \mathrm{z} 378.1\left[\mathrm{M}+\mathrm{H}^{+}\right]$. 


\section{Derivatization of planar cellulose supports.}

Representative planar cellulose membrane amination protocol. Dots were marked on a $14 \mathrm{~cm} \times 18 \mathrm{~cm}$ sheet of Whatman $1 \mathrm{Chr}$ paper at distances $1.4 \mathrm{~cm}$ apart using a \#2 lead pencil. The sheet was immersed in $100 \mathrm{~mL}$ of $10 \%$ TFA in $\mathrm{CH}_{2} \mathrm{Cl}_{2}$ for $10 \mathrm{~min}$ in a covered $2.6 \mathrm{~L}$ Pyrex dish. This acid wash serves as a cellulose preactivation step and is believed to increase the surface area of the cellulose available for functionalization. ${ }^{6}$ The sheet was washed by adding 60 $\mathrm{mL}$ of $\mathrm{CH}_{2} \mathrm{Cl}_{2}$, allowing it soak for $5 \mathrm{~min}$, then decanting the $\mathrm{CH}_{2} \mathrm{Cl}_{2}$. This process was repeated, after which the membrane was dried under a stream of air for $10 \mathrm{~min}$. The sheet was next immersed in $50 \mathrm{~mL}$ of $2 \mathrm{M}$ tosyl chloride in pyridine for $2 \mathrm{~h}$. The paper was washed by immersion in three consecutive baths of EtOH $(100 \mathrm{~mL}, 5 \mathrm{~min}$ each $)$ and dried under a stream of $\mathrm{N}_{2}$.

To install the flexible amine space, the tosylated cellulose paper was immersed in $60 \mathrm{~mL}$ of neat 4,7,10-trioxa-1,13-tridecanediamine ${ }^{7}$ and placed on the rotating platform of the Milestone microwave reactor. The dish was covered with a second Pyrex dish. The sheet was irradiated for $15 \mathrm{~min}$ at $400 \mathrm{~W}$. The amine solution was carefully decanted from the paper. The paper was washed by adding then decanting $70 \mathrm{~mL}$ portions of DMF, $\mathrm{EtOH}, 1.0 \mathrm{~N} \mathrm{NaOH}, \mathrm{H}_{2} \mathrm{O}, \mathrm{EtOH}(2 \mathrm{x})$, and $\mathrm{CH}_{2} \mathrm{Cl}_{2}$ (5 min in each bath). The amino paper was then dried under a stream of $\mathrm{N}_{2}$.

Representative Fmoc quantitation protocol on cellulose supports. A spot $(6 \mathrm{~mm}$ diameter) was punched from amino cellulose support using a desktop hole punch and immersed in $200 \mu \mathrm{L}$ of $0.60 \mathrm{M}$ Fmoc-OSu in DMF for $2 \mathrm{~h}$. The spot was washed with $10 \mathrm{~mL}$ of EtOH, 10 $\mathrm{mL}$ of acetone, and $10 \mathrm{~mL}$ of $\mathrm{CH}_{2} \mathrm{Cl}_{2}$. After drying under a stream of $\mathrm{N}_{2}, 960 \mu \mathrm{L}$ of DMF was added followed by $40 \mu \mathrm{L}$ of 1,8-diazabicyclo[5.4.0]undec-7-ene (DBU). The mixture was swirled for $30 \mathrm{sec}$ and then allowed to stand for $15 \mathrm{~min}$. The mixture was swirled again for 30 sec, then $250 \mu \mathrm{L}$ of this solution was removed and diluted with $1.0 \mathrm{~mL}$ of DMF. The solution was swirled again for $30 \mathrm{sec}$. The absorbance was read at $296 \mathrm{~nm}\left(\varepsilon_{296}=9500 \mathrm{M}^{-1} \mathrm{~cm}^{-1}\right)$ in a quartz cuvette. The value was multiplied by 5 to account for the dilution. ${ }^{8}$ Loadings of $1.0-10$ $\mu \mathrm{mol} / \mathrm{cm}^{2}$ were obtained using this method. Longer tosylation reaction times gave higher levels of functionalization (e.g., $2 \mathrm{~h}=4.0 \mu \mathrm{mol} / \mathrm{cm}^{2}, 12 \mathrm{~h}=10 \mu \mathrm{mol} / \mathrm{cm}^{2}$ ).

Representative Wang-linker installation procedure on amino cellulose support. 4formylphenoxyacetic acid $(5.40 \mathrm{~g}, 30.0 \mathrm{mmol})$, diisopropylcarbodiimide (DIC, $4.7 \mathrm{~mL}, 30.0$ $\mathrm{mmol}), N$-hydroxysuccinimide $(3.45 \mathrm{~g}, 30.0 \mathrm{mmol}), \mathrm{NEt}_{3}(4.2 \mathrm{~mL}, 30.0 \mathrm{mmol})$, and DMF (50 $\mathrm{mL}$ ) were combined in a $2.6 \mathrm{~L}$ Pyrex dish. The dish was covered and swirled for $30 \mathrm{~min}$ at room temperature. A $14 \mathrm{~cm} \times 18 \mathrm{~cm}$ sheet of amino cellulose support was added. The dish was covered again and the mixture was swirled at room temperature for $2 \mathrm{~h}$. The coupling solution was decanted. ${ }^{9}$ The paper was washed by adding then decanting $70 \mathrm{~mL}$ portions of DMF (2x), EtOH (2x), and $\mathrm{CH}_{2} \mathrm{Cl}_{2}$ (5 min in each bath). The aldehyde-derived paper was then dried under a stream of $\mathrm{N}_{2}$.

A $100 \mathrm{~mL}$ aliquot of $1.0 \mathrm{M} \mathrm{NaBH}_{4}$ in $1.0 \mathrm{M}$ aq. $\mathrm{NaOH}$ was added to the aldehyde-derived support. The mixture was swirled for 20 min, after which the $\mathrm{NaBH}_{4}$ solution was decanted. The paper was then washed by adding then decanting $70 \mathrm{~mL}$ portions of $\mathrm{H}_{2} \mathrm{O}(2 \mathrm{x}), \mathrm{EtOH}(2 \mathrm{x})$, and $\mathrm{CH}_{2} \mathrm{Cl}_{2}$ (5 min in each bath). The benzyl alcohol paper was dried under a stream of $\mathrm{N}_{2}$. To approximate the linker loading, the amount of residual amine was measured by Fmoc quantitation as described above, except a 2-3-fold dilution was used instead. Residual amine loadings are $c a .600-800 \mathrm{nmol} / \mathrm{cm}^{2}$. 
Representative Wang-linker activation protocol. Immediately prior to use, a $14 \mathrm{~cm} \times 18$ $\mathrm{cm}$ sheet of benzyl alcohol paper was activated by submerging it in $50 \mathrm{~mL}$ of a $4.0 \mathrm{M}$ solution of tosyl chloride in dry DMF and swirled for $30 \mathrm{~min}$ on an orbital shaker. The paper was washed by immersion and swirling in dry $\mathrm{CH}_{2} \mathrm{Cl}_{2}(70 \mathrm{~mL}, 3$ x $2 \mathrm{~min})$. The benzyl chloride-derived paper was then dried by a stream of $\mathrm{N}_{2}$ for 10 min prior to the next step.

\section{Synthesis of chalcone macroarray 1.}

Coupling of hydroxyacetophenones to activated planar support. A $2.0 \mathrm{M}$ coupling solution of the various hydroxyacetophenones solutions were prepared by adding an equal volume of a $4.0 \mathrm{M}$ solution of $\mathrm{KO} t \mathrm{Bu}$ in anhydrous $\mathrm{DMF}$ to a $4.0 \mathrm{M}$ solution of substituted hydroxyacetophenone in anhydrous DMF. Twelve 3.0 $\mu \mathrm{L}$ aliquots of each solution was applied to activated Wang-linker derived cellulose as twelve spots at distances $1.4 \mathrm{~cm}$ apart along three rows $(\mathbf{A}-\mathbf{C})$ :

Row A: 4'-hydroxyacetophenone

Row B: 3'-hydroxyacetophenone

Row C: acetovanillone

The spotted planar support was subjected to microwave irradiation in a Pyrex dish at $500 \mathrm{~W}$ for $10 \mathrm{~min}$ in the Milestone microwave reactor. The support was next washed in a Pyrex dish by adding then decanting $70 \mathrm{~mL}$ portions of $1 \mathrm{~N} \mathrm{NaOH}, \mathrm{H}_{2} \mathrm{O}(2 \mathrm{x}), \mathrm{EtOH}(2 \mathrm{x})$, and $\mathrm{CH}_{2} \mathrm{Cl}_{2}(5 \mathrm{~min}$ in each bath). The acetophenone-derived support was dried under a stream of $\mathrm{N}_{2}$ (for $10 \mathrm{~min}$ ) prior to the next step.

Claisen-Schmidt condensation on planar support. The following benzaldehyde solutions were prepared and three aliquots were spotted onto acetophenones-derived support at distances $1.4 \mathrm{~cm}$ apart down the corresponding columns (1-12):

Column 1: $\quad 6.0 \mu \mathrm{L}$ of $1.0 \mathrm{M}$ benzaldehyde in $1.5 \mathrm{~N} \mathrm{NaOH}$ in $50 \%$ aq. EtOH.

Column 2: $\quad 6.0 \mu \mathrm{L}$ of $0.5 \mathrm{M}$ 2-fluorobenzaldehyde in $1.5 \mathrm{~N} \mathrm{NaOH}$ in $50 \%$ aq. EtOH.

Column 3: $\quad 6.0 \mu \mathrm{L}$ of $1.0 \mathrm{M}$ 3-fluorobenzaldehyde in $1.5 \mathrm{~N} \mathrm{NaOH}$ in $50 \%$ aq. EtOH.

Column 4: $\quad 6.0 \mu \mathrm{L}$ of $1.0 \mathrm{M}$ 4-fluorobenzaldehyde in $1.5 \mathrm{~N} \mathrm{NaOH}$ in $50 \%$ aq. EtOH.

Column 5: $\quad 6.0 \mu \mathrm{L}$ of $0.5 \mathrm{M} 3$-bromobenzaldehyde in $1.5 \mathrm{~N} \mathrm{NaOH}$ in $50 \%$ aq. EtOH.

Column 6: $\quad 6.0 \mu \mathrm{L}$ of $0.5 \mathrm{M}$ 4-bromobenzaldehyde in $1.5 \mathrm{~N} \mathrm{NaOH}$ in $50 \%$ aq. EtOH.

Column 7: $\quad 6.0 \mu \mathrm{L}$ of $0.5 \mathrm{M}$ 4-chlorobenzaldehyde in $1.5 \mathrm{~N} \mathrm{NaOH}$ in $50 \%$ aq. EtOH.

Column 8: $\quad 6.0 \mu \mathrm{L}$ of $1.0 \mathrm{M} \mathrm{3,4-difluorobenzaldehyde} \mathrm{in} 1.5 \mathrm{~N} \mathrm{NaOH}$ in $50 \%$ aq. $\mathrm{EtOH}$.

Column 9: $\quad 6.0 \mu \mathrm{L}$ of $0.5 \mathrm{M} \mathrm{m}$-anisaldehyde in $1.5 \mathrm{~N} \mathrm{NaOH}$ in $50 \%$ aq. EtOH.

Column 10: $\quad 6.0 \mu \mathrm{L}$ of $1.0 \mathrm{M}$ p-anisaldehyde in $1.5 \mathrm{~N} \mathrm{NaOH}$ in $50 \%$ aq. EtOH.

Column 11: $\quad 6.0 \mu \mathrm{L}$ of $1.0 \mathrm{M} 3$-chlorobenzaldehyde in $1.5 \mathrm{~N} \mathrm{NaOH}$ in $50 \%$ aq. EtOH.

Column 12: $\quad 6.0 \mu \mathrm{L}$ of $1.0 \mathrm{M}$ 4-dimethylaminobenzaldehyde in warm ethylene glycol.

To column 12, 8.0 $\mu \mathrm{L}$ of $5.0 \mathrm{M} \mathrm{KO} t \mathrm{Bu}$ in ethylene glycol was added. (Since 4dimethylaminobenzaldehyde was insoluble in high concentrations of base, the solution of aldehyde and the solution of the base were added separately). The spotted support was placed in 
a Pyrex dish in the Milestone microwave reactor and irradiated at $400 \mathrm{~W}$ for $10 \mathrm{~min}$. Columns 112 were then respotted with the benzaldehyde solutions and irradiated a second time in the Milestone microwave reactor at $400 \mathrm{~W}$ for $10 \mathrm{~min}$. The support was next washed in a Pyrex dish by adding then decanting $70 \mathrm{~mL}$ portions of $1 \%$ aq. $\mathrm{AcOH}, \mathrm{DMSO}, \mathrm{EtOH}(2 \mathrm{x})$, and $\mathrm{CH}_{2} \mathrm{Cl}_{2}(5$ min in each bath). The chalcone-derived support was dried under a stream of $\mathrm{N}_{2}$.

\section{Synthesis of fluorescent dye macroarrays 2 and 5.}

Cyanopyridine macroarray (2): $\mathrm{KO} t \mathrm{Bu}(1.234 \mathrm{~g}, 11.0 \mathrm{mmol})$ was suspended in $5.0 \mathrm{~mL}$ of acetonitrile in a $20 \mathrm{~mL}$ vial. The mixture was capped with a Teflon-lined cap and sonicated in an ultrasound bath (Branson model \# 1510R-MT) for $50 \mathrm{~min}$. After sonication, $6.0 \mu \mathrm{L}$ aliquots of the mixture were applied to each spot on chalcone array 1 . The membrane was allowed to stand at room temperature for $10 \mathrm{~min}$. The spotting and standing steps were repeated three more times. The membrane was washed in a Pyrex dish by adding then decanting $70 \mathrm{~mL}$ portions of DMSO, $1 \%$ aq. acetic acid, $\mathrm{EtOH}(2 \mathrm{x})$, and $\mathrm{CH}_{2} \mathrm{Cl}_{2}$ (5 min in each bath). The cyanopyridine array (2) was then dried under a stream of $\mathrm{N}_{2}$.

Deazalumazine macroarray (5): 6-amino-2,3-dimethyl uracil $(4,77.6 \mathrm{mg}, 0.5 \mathrm{mmol})$ was dissolved in $1.5 \mathrm{~mL}$ of DMSO and $0.5 \mathrm{~mL}$ of $1.0 \mathrm{~N}$ aq. $\mathrm{NaOH}$. A $6.0 \mu \mathrm{L}$ aliquot of this solution was applied to each spot on chalcone array 1. The support was placed in a Pyrex dish in the Milestone microwave reactor and irradiated at $400 \mathrm{~W}$ for $10 \mathrm{~min}$. The spotting and microwave irradiation steps were repeated three more times. The membrane was washed in a Pyrex dish by adding then decanting $70 \mathrm{~mL}$ portions of DMSO, $1 \%$ aq. acetic acid, EtOH (2x), and $\mathrm{CH}_{2} \mathrm{Cl}_{2}(5$ min in each bath). The deazalumazine array (5) was then dried under a stream of $\mathrm{N}_{2}$.

Vapor-phase cleavage of dye compounds from macroarrays. This cleavage method is a modified version of that reported by Scharn et al. ${ }^{10}$ Compound spots were punched out from the macroarray with a desktop hole-punch and placed in individual vials. TFA (10 mL) was added to the bottom of a glass vacuum dessicator $(26 \mathrm{~cm}$ diameter, $20 \mathrm{~cm}$ tall). A Petri dish $(14.5 \mathrm{~cm}$ diameter) was placed on a ceramic platform above the TFA, and the vials containing the macroarray members were placed on this dish. The dessicator was evacuated to 60 mbar for 10 min and then sealed for $50 \mathrm{~min}$. The seal was broken, and the vials then were removed from of the dessicator. The vials were allowed to stand in a fume hood at room temperature for $30 \mathrm{~min}$, after which $1.0 \mathrm{~mL}$ of EtOH was added to each vial. After swirling for $15 \mathrm{~min}, 0.5 \mathrm{~mL}$ of each solution was removed for LC-MS analyses and placed in separate glass vials. The remaining 0.5 $\mathrm{mL}$ portions were used for spectral characterization of the macroarray members.

LC-MS analysis of dye compounds. The EtOH solutions were concentrated under reduced pressure using a SpeedVac centrifuge system. The resulting residues were dissolved in $150 \mu \mathrm{L}$ of $50 \%$ aqueous acetonitrile, filtered through a cotton plug, and analyzed by LC-MS. HPLC: conditions as described above, solvent gradient 20-95\% B in 8 min followed by 4 min at $95 \%$ B. MS: (ESI) positive ion mode was used for all samples. Relative conversions (in comparison to acetophenone and chalcone starting materials) and compound purities were determined by integration of peaks in the HPLC trace at $254 \mathrm{~nm}$ using the Shimadzu LCMSolution software package. 


\section{Spectral characterization methods.}

Screening of macroarrays and fluorophore solutions using a handheld UV-lamp. Dried macroarrays or solutions (in $4.0 \mathrm{~mL}$ Pyrex vials) were placed under a handheld UV-lamp (C. Entela Mineralight ${ }^{\circledR}$ Lamp; model UVGL-58). Irradiation of the macroarrays at $254 \mathrm{~nm}$ yielded the highest contrast between spots. Solutions in Pyrex vials were irradiated at $366 \mathrm{~nm}$, as Pyrex blocks shortwave UV $(254 \mathrm{~nm})$. A digital camera (Canon PowerShot SD500) was used to photograph the arrays and solutions, using the "daylight white balance" setting. On occasion, recording the irradiated samples in the "filming mode" and copying a specific frame using Microsoft Windows Media Player ${ }^{\circledR}$ (V 10.0) provided the most accurate photographs.

Standard preparation. A stock solution of the fluorescence standard coumarin $120(1 \mathrm{mg}$ in $4.0 \mathrm{~mL}$ of EtOH) was diluted with EtOH until the absorbance at $340 \mathrm{~nm}$ was between 0.05 and $0.01 .^{11}$ The fluorescence spectra were recorded using an excitation wavelength of $340 \mathrm{~nm}$. The fluorescence emission was integrated with Microsoft Excel. This was repeated three times at different optical densities.

Evaluation of macroarray compounds. Each cleaved spot sample was diluted with EtOH until the absorbance at $340 \mathrm{~nm}$ was between 0.05 and 0.01 . The fluorescence spectra were recorded with an excitation wavelength of $340 \mathrm{~nm}$. The peak was integrated using Microsoft Excel. This process was repeated three times at different optical densities. The integrated intensity was plotted against the absorbance of each sample. The slopes of the resulting lines were calculated using Microsoft Excel. The quantum yield was then determined by the following equation: ${ }^{1}$

$$
\phi_{\text {sample }}=\phi_{\text {std }} \frac{\left(\text { Slope }_{\text {sample }}\right)}{\left(\text { Slope }_{\text {std }}\right)} \frac{\left(\eta_{\text {sample }}^{2}\right)}{\left(\eta_{\text {std }}^{2}\right)}
$$

Evaluation of solution-phase standards. Stock solutions of fluorophores prepared by solution-phase synthesis were made at the approximate concentration of $1 \mathrm{mg}$ in $4.0 \mathrm{~mL}$ of solvent. Aliquots of the stock solutions were diluted until the absorbance at $340 \mathrm{~nm}$ was between 0.05 and 0.01 . The fluorescence spectra were recorded with an excitation wavelength of $340 \mathrm{~nm}$. Quantum yields were calculated as described above. ${ }^{1}$ 


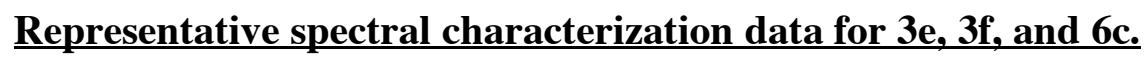

Characterization of cyanopyridine $3 \mathrm{e}$.<smiles>COc1ccc(-c2cc(-c3ccc(O)cc3)nc([N+](=O)[O-])c2C#N)cc1</smiles>

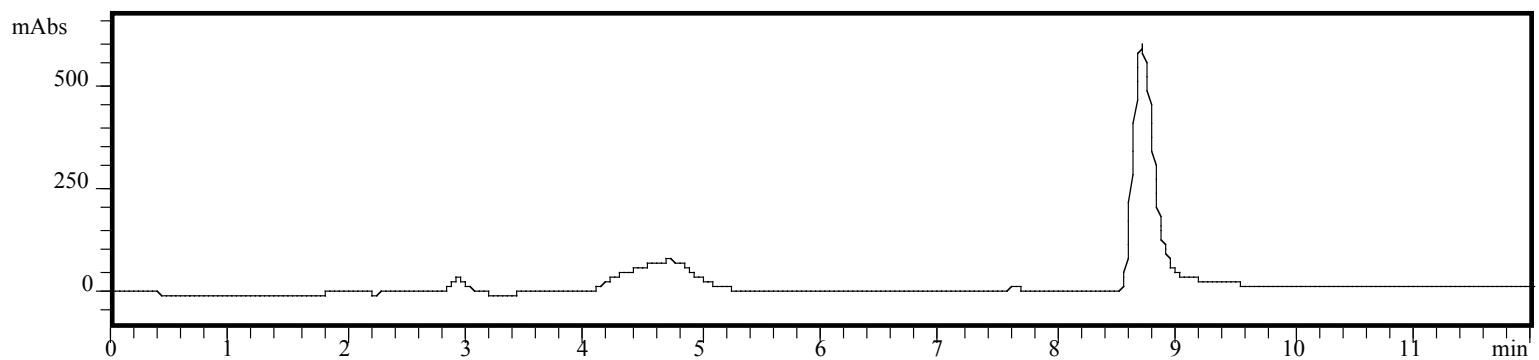

HPLC trace of crude $\mathbf{3 e}$ obtained from macroarray $(254 \mathrm{~nm})$.

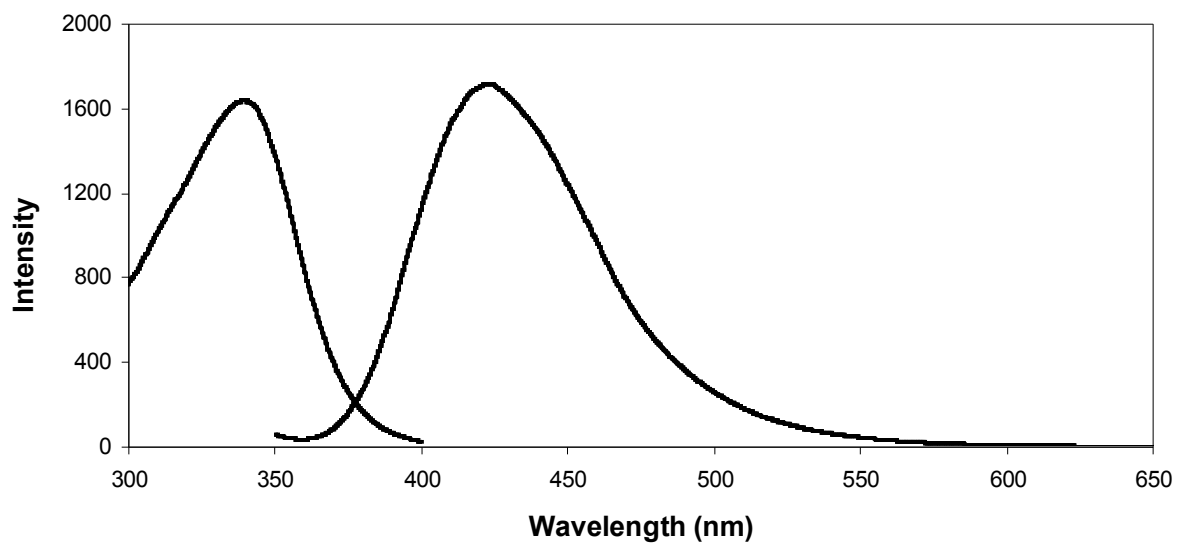

Excitation and emission spectra of crude 3e obtained from macroarray (in EtOH).

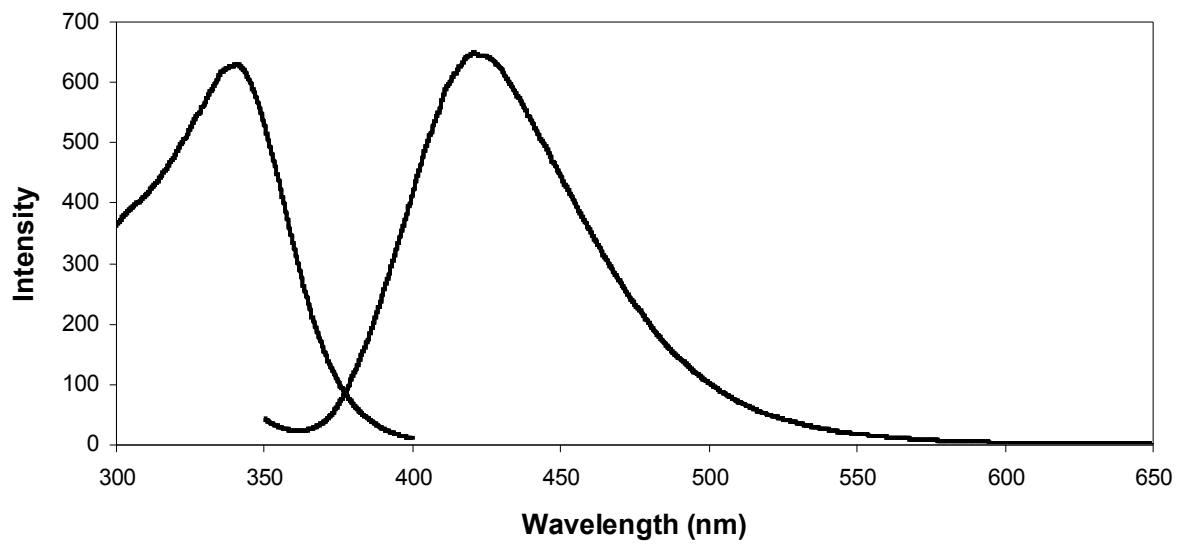

Excitation and emission spectra of purified 3e obtained from solution-phase synthesis (in EtOH). 


\section{Characterization of cyanopyridine $3 \mathrm{f}$.}<smiles>CC(C)(C)Oc1ccc(-c2cc(-c3ccc(O)cc3)nc([N+](=O)[O-])c2C#N)cc1</smiles>

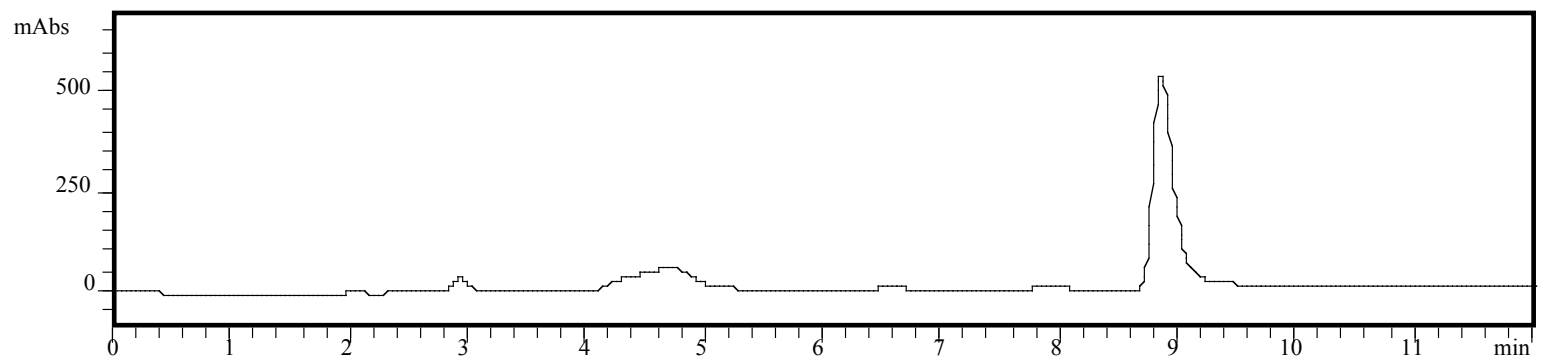

HPLC trace of crude $\mathbf{3 f}$ obtained from macroarray $(254 \mathrm{~nm})$.

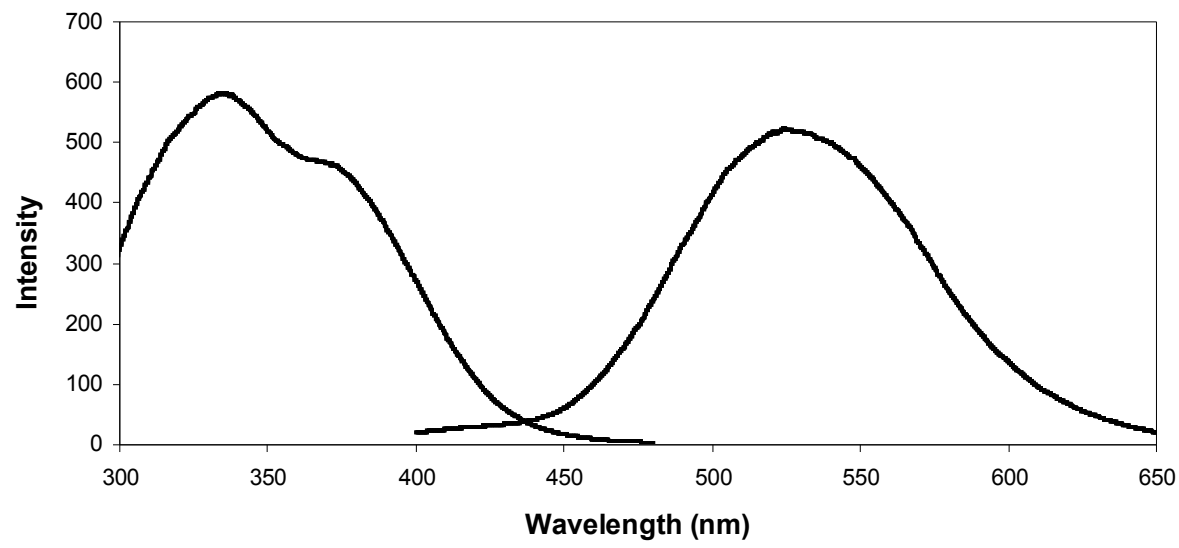

Excitation and emission spectra of crude $\mathbf{3 f}$ obtained from macroarray (in EtOH).

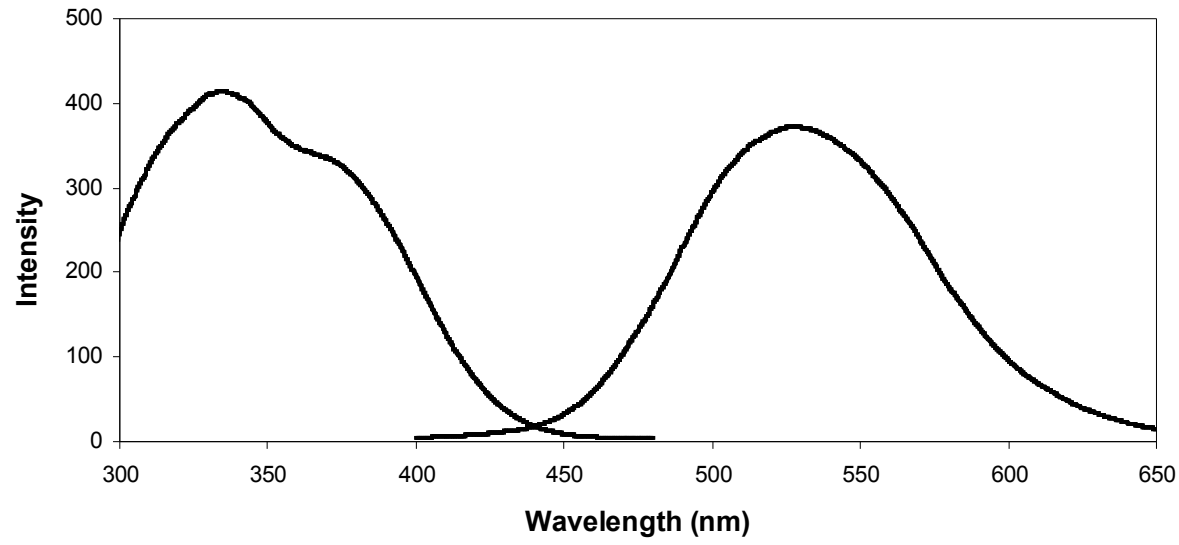

Excitation and emission spectra of purified 3f obtained from solution-phase synthesis (in EtOH). 


\section{Characterization of deazalumazine $6 c$.}
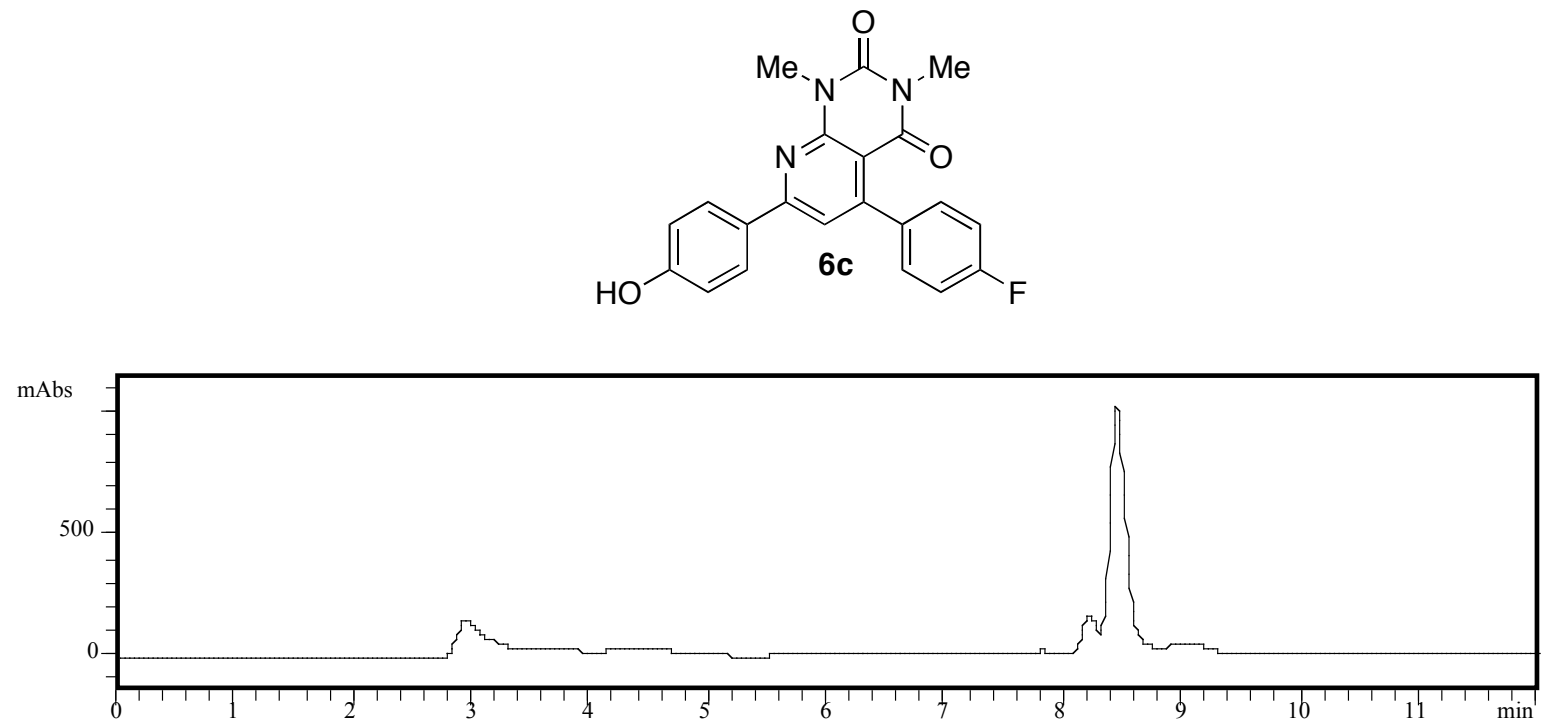

HPLC trace of crude $\mathbf{6 c}$ obtained from macroarray (254 nm).

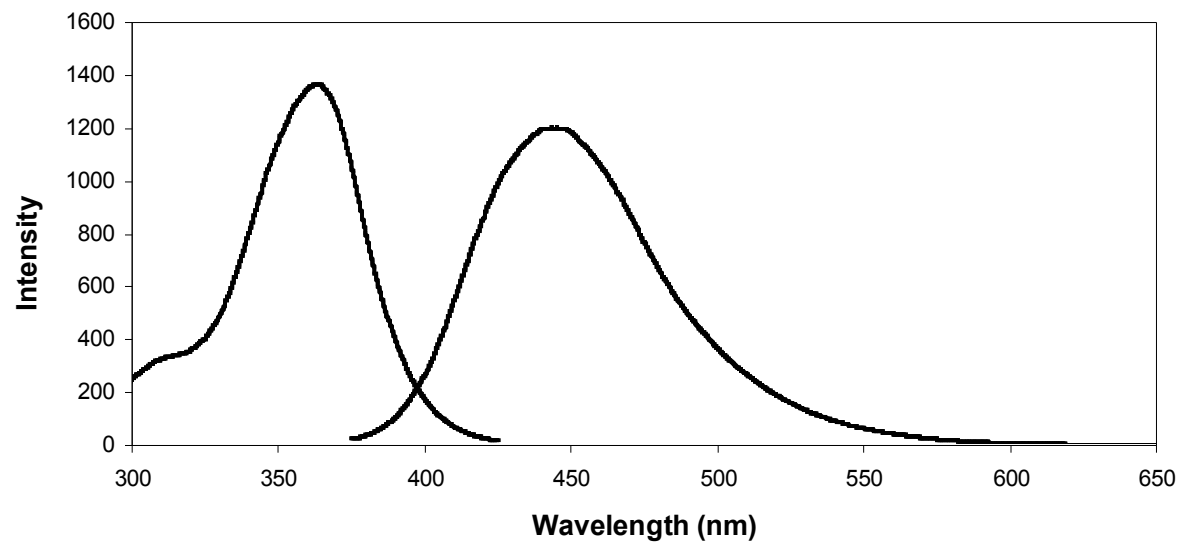

Excitation and emission spectra of crude $\mathbf{6 c}$ obtained from macroarray (in EtOH).

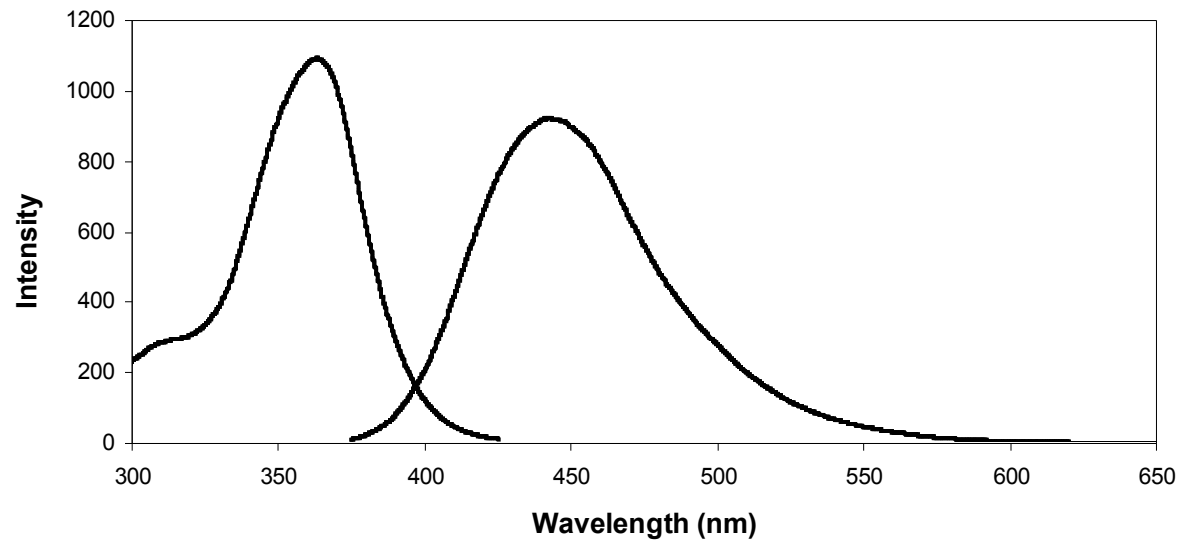

Excitation and emission spectra of purified $\mathbf{6 c}$ obtained from solution-phase synthesis (in EtOH). 


\section{LC-MS analysis of cyanopyridines (3) cleaved from macroarray 2.}<smiles>[R]c1cccc(-c2cc(-c3cccc([R])c3)c(C#N)c(C)n2)c1</smiles>

\begin{tabular}{|c|c|c|c|c|c|c|}
\hline entry $^{a}$ & $\mathrm{R}^{1}$ & $\mathrm{R}^{2}$ & $\begin{array}{l}\text { retention time } \\
(\text { min) }\end{array}$ & $\begin{array}{l}\text { calc. } \\
\text { mass }\end{array}$ & $\begin{array}{l}\text { obs. } \\
\text { mass }\end{array}$ & $\begin{array}{l}\text { purity } \\
(\%)^{b}\end{array}$ \\
\hline $\mathbf{3 a}$ & $4-\mathrm{OH}$ & $\mathrm{H}$ & 8.713 & 286.1 & $286.9[\mathrm{M}+\mathrm{H}]^{+}$ & 81 \\
\hline $3 \mathbf{b}$ & $4-\mathrm{OH}$ & $2-\mathrm{F}$ & 8.629 & 304.1 & $304.9[\mathrm{M}+\mathrm{H}]^{+}$ & 81 \\
\hline $3 c$ & $4-\mathrm{OH}$ & $4-\mathrm{F}$ & 8.839 & 304.1 & $304.9[\mathrm{M}+\mathrm{H}]^{+}$ & 83 \\
\hline 3d & $4-\mathrm{OH}$ & $4-\mathrm{Br}$ & 9.553 & 364.0 & $365.0[\mathrm{M}+\mathrm{H}]^{+}$ & 82 \\
\hline $3 e$ & $4-\mathrm{OH}$ & 4-OMe & 8.869 & 316.1 & $316.9[\mathrm{M}+\mathrm{H}]^{+}$ & 70 \\
\hline \multirow[t]{2}{*}{$3 f$} & $4-\mathrm{OH}$ & $4-\mathrm{NMe}_{2}$ & 8.846 & 329.2 & $329.9[\mathrm{M}+\mathrm{H}]^{+}$ & 74 \\
\hline & $4-\mathrm{OH}$ & $3-\mathrm{F}$ & 8.859 & 304.1 & $304.9[\mathrm{M}+\mathrm{H}]^{+}$ & 78 \\
\hline $3 g$ & 3-OMe, 4-OH, & $\mathrm{H}$ & 8.838 & 316.1 & $316.9[\mathrm{M}+\mathrm{H}]^{+}$ & 74 \\
\hline $3 h$ & 3-OMe, 4-OH, & $3-\mathrm{OMe}$ & 8.932 & 346.1 & $346.9[\mathrm{M}+\mathrm{H}]^{+}$ & 89 \\
\hline $3 \mathbf{i}$ & 3-OMe, 4-OH, & $4-\mathrm{F}$ & 8.952 & 334.1 & $335.0[\mathrm{M}+\mathrm{H}]^{+}$ & 71 \\
\hline $3 \mathbf{j}$ & 3-OMe, 4-OH, & $4-\mathrm{Br}$ & 9.698 & 394.0 & $394.9[\mathrm{M}+\mathrm{H}]^{+}$ & 70 \\
\hline $3 \mathbf{k}$ & 3-OMe, 4-OH, & 4-OMe & 8.852 & 346.1 & $346.9[\mathrm{M}+\mathrm{H}]^{+}$ & 78 \\
\hline \multirow[t]{3}{*}{31} & 3-OMe, 4-OH, & $4-\mathrm{NMe}_{2}$ & 9.020 & 359.2 & $359.9[\mathrm{M}+\mathrm{H}]^{+}$ & 87 \\
\hline & 3-OMe, 4-OH, & $2-\mathrm{F}$ & 8.792 & 334.1 & $334.9[\mathrm{M}+\mathrm{H}]^{+}$ & 91 \\
\hline & 3-OMe, 4-OH, & $3-\mathrm{F}$ & 9.030 & 334.1 & $334.9[\mathrm{M}+\mathrm{H}]^{+}$ & 89 \\
\hline \multirow[t]{5}{*}{$3 \mathrm{~m}$} & $3-\mathrm{OH}$ & 4-OMe & 8.739 & 316.1 & $317.0[\mathrm{M}+\mathrm{H}]^{+}$ & 84 \\
\hline & $3-\mathrm{OH}$ & $3-\mathrm{Br}$ & 9.496 & 364.0 & $364.8[\mathrm{M}+\mathrm{H}]^{+}$ & 77 \\
\hline & $3-\mathrm{OH}$ & $4-\mathrm{Br}$ & 9.617 & 364.0 & $364.8[\mathrm{M}+\mathrm{H}]^{+}$ & 86 \\
\hline & $3-\mathrm{OH}$ & $3-\mathrm{Cl}$ & 9.476 & 320.1 & $320.9[\mathrm{M}+\mathrm{H}]^{+}$ & 85 \\
\hline & $3-\mathrm{OH}$ & $3-F, 4-F$ & 9.097 & 322.1 & $322.9[\mathrm{M}+\mathrm{H}]^{+}$ & 83 \\
\hline
\end{tabular}

${ }^{a}$ Library members without compound numbers represent compounds that were omitted from the text for the sake of brevity. ${ }^{b}$ Determined by HPLC integration at $254 \mathrm{~nm}$. 


\section{LC-MS analysis of deazalumazines (6) cleaved from macroarray 5.}<smiles>[H][R]1cccc(-c2cc(-c3ccccc3)c3c(=O)n(C)c(=O)n(C)c3n2)c1</smiles>

\begin{tabular}{|c|c|c|c|c|c|c|}
\hline entry $^{a}$ & $\mathrm{R}^{1}$ & $\mathrm{R}^{2}$ & $\begin{array}{l}\text { retention time } \\
(\mathrm{min})\end{array}$ & $\begin{array}{l}\text { calc. } \\
\text { mass }\end{array}$ & $\begin{array}{l}\text { obs. } \\
\text { mass }\end{array}$ & $\begin{array}{l}\text { purity } \\
(\%)^{b}\end{array}$ \\
\hline $6 a$ & $4-\mathrm{OH}$ & $\mathrm{H}$ & 8.269 & 359.1 & $360.1[\mathrm{M}+\mathrm{H}]^{+}$ & 77 \\
\hline $6 \mathrm{~b}$ & $4-\mathrm{OH}$ & $2-\mathrm{F}$ & 8.232 & 377.1 & $377.9[\mathrm{M}+\mathrm{H}]^{+}$ & 79 \\
\hline $6 c$ & $4-\mathrm{OH}$ & $4-\mathrm{F}$ & 8.433 & 377.1 & $377.9[\mathrm{M}+\mathrm{H}]^{+}$ & 78 \\
\hline 6d & $4-\mathrm{OH}$ & $4-\mathrm{Br}$ & 9.129 & 437.0 & $438.0[\mathrm{M}+\mathrm{H}]^{+}$ & 78 \\
\hline \multirow[t]{5}{*}{$6 e$} & $4-\mathrm{OH}$ & 4-OMe & 8.210 & 389.1 & $390.0[\mathrm{M}+\mathrm{H}]^{+}$ & 82 \\
\hline & $4-\mathrm{OH}$ & $4-\mathrm{Cl}$ & 9.014 & 393.1 & $393.8[\mathrm{M}+\mathrm{H}]^{+}$ & 79 \\
\hline & $4-\mathrm{OH}$ & $4-\mathrm{Br}$ & 9.166 & 437.0 & $438.0[\mathrm{M}+\mathrm{H}]^{+}$ & 87 \\
\hline & $4-\mathrm{OH}$ & $3-\mathrm{F}$ & 8.433 & 377.1 & $378.1[\mathrm{M}+\mathrm{H}]^{+}$ & 87 \\
\hline & $4-\mathrm{OH}$ & $3-\mathrm{Cl}$ & 8.955 & 393.1 & $393.9[\mathrm{M}+\mathrm{H}]^{+}$ & 86 \\
\hline $6 g$ & 3-OMe, 4-OH & $\mathrm{H}$ & 8.440 & 389.1 & $390.1[\mathrm{M}+\mathrm{H}]^{+}$ & 82 \\
\hline $6 h$ & 3-OMe, 4-OH & 3-OMe & 8.455 & 419.2 & $419.9[\mathrm{M}+\mathrm{H}]^{+}$ & 78 \\
\hline $\mathbf{6 i}$ & 3-OMe, 4-OH & $4-\mathrm{F}$ & 8.573 & 407.1 & $408.0[\mathrm{M}+\mathrm{H}]^{+}$ & 76 \\
\hline $\mathbf{6 j}$ & 3-OMe, 4-OH & $4-\mathrm{Br}$ & 9.371 & 467.1 & $468.0[\mathrm{M}+\mathrm{H}]^{+}$ & 76 \\
\hline $6 \mathbf{k}$ & 3-OMe, 4-OH & 4-OMe & 8.358 & 419.2 & $420.1[\mathrm{M}+\mathrm{H}]^{+}$ & 77 \\
\hline $6 r$ & $3-\mathrm{OH}$ & $4-\mathrm{F}$ & 8.594 & 377.1 & $377.9[\mathrm{M}+\mathrm{H}]^{+}$ & 87 \\
\hline \multirow[t]{4}{*}{$6 s$} & $3-\mathrm{OH}$ & $4-\mathrm{Cl}$ & 9.182 & 393.1 & $394.1[\mathrm{M}+\mathrm{H}]^{+}$ & 84 \\
\hline & $3-\mathrm{OH}$ & $3-\mathrm{Br}$ & 9.250 & 437.0 & $437.9[\mathrm{M}+\mathrm{H}]^{+}$ & 95 \\
\hline & $3-\mathrm{OH}$ & $4-\mathrm{Br}$ & 9.319 & 437.0 & $437.9[\mathrm{M}+\mathrm{H}]^{+}$ & 86 \\
\hline & $3-\mathrm{OH}$ & $\mathrm{H}$ & 8.436 & 359.1 & $360.1[\mathrm{M}+\mathrm{H}]^{+}$ & 79 \\
\hline
\end{tabular}

${ }^{a}$ Library members without compound numbers represent compounds that were omitted from the text for the sake of brevity. ${ }^{b}$ Determined by HPLC integration at $254 \mathrm{~nm}$. 


\section{Spectral data for cyanopyridines 3.}<smiles>[R]c1cccc(-c2cc(-c3ccccc3)c(C#N)c(C)n2)c1</smiles>

\begin{tabular}{|c|c|c|c|c|c|c|c|c|c|c|c|}
\hline entry $^{a}$ & $\mathrm{R}^{1}$ & $\mathrm{R}^{2}$ & $\lambda_{\mathrm{ex}}$ & $\lambda_{\mathrm{em}}$ & $\phi_{\mathrm{f}}^{b}$ & entry $^{a}$ & $\mathrm{R}^{1}$ & $\mathrm{R}^{2}$ & $\lambda_{\mathrm{ex}}$ & $\lambda_{\mathrm{em}}$ & $\phi_{\mathrm{f}}^{b}$ \\
\hline 3a & $4-\mathrm{OH}$ & $\mathrm{H}$ & 341 & 433 & 0.07 & 3d & $4-\mathrm{OH}$ & $4-\mathrm{Br}$ & 342 & 440 & 0.04 \\
\hline \multirow[t]{2}{*}{$3 \mathbf{b}$} & $4-\mathrm{OH}$ & $2-\mathrm{F}$ & 342 & 430 & 0.06 & & $4-\mathrm{OH}$ & $4-\mathrm{Cl}$ & 343 & 443 & 0.04 \\
\hline & $4-\mathrm{OH}$ & $3-\mathrm{F}$ & 334 & 445 & 0.04 & & $4-\mathrm{OH}$ & $3-\mathrm{Cl}$ & 343 & 446 & 0.03 \\
\hline \multirow[t]{3}{*}{$3 c$} & $4-\mathrm{OH}$ & $4-\mathrm{F}$ & 341 & 430 & 0.07 & & $4-\mathrm{OH}$ & 3-OMe & 341 & 433 & 0.05 \\
\hline & $4-\mathrm{OH}$ & $3 F, 4-F$ & 343 & 441 & 0.03 & $3 \mathbf{e}$ & $4-\mathrm{OH}$ & 4-OMe & 339 & 423 & 0.12 \\
\hline & $4-\mathrm{OH}$ & $3-\mathrm{Br}$ & 344 & 445 & 0.03 & $3 f$ & $4-\mathrm{OH}$ & 4- $\mathrm{NMe}_{2}$ & 366 & 524 & 0.09 \\
\hline \multirow[t]{3}{*}{$3 g$} & $3-\mathrm{OMe}, 4-\mathrm{OH}$ & $\mathrm{H}$ & 351 & 469 & 0.03 & $3 \mathbf{j}$ & 3-OMe, 4-OH & $4-\mathrm{Br}$ & 352 & 475 & 0.02 \\
\hline & 3-OMe, 4-OH & $2-\mathrm{F}$ & 351 & 465 & 0.02 & & 3-OMe, 4-OH & $4-\mathrm{Cl}$ & 351 & 474 & 0.02 \\
\hline & 3-OMe, 4-OH & $3-\mathrm{F}$ & 356 & 476 & 0.01 & & 3-OMe, 4-OH & $3-\mathrm{Cl}$ & 355 & 485 & 0.01 \\
\hline \multirow[t]{9}{*}{$3 \mathbf{i}$} & 3-OMe, 4-OH & $4-\mathrm{F}$ & 351 & 466 & 0.04 & $3 \mathrm{~h}$ & 3-OMe, 4-OH & 3-OMe & 351 & 473 & 0.03 \\
\hline & 3-OMe, 4-OH & $3 F, 4-F$ & 355 & 471 & 0.02 & $3 \mathbf{k}$ & 3-OMe, 4-OH & 4-OMe & 351 & 461 & 0.06 \\
\hline & 3-OMe, 4-OH & $3-\mathrm{Br}$ & 353 & 477 & 0.02 & 31 & 3-OMe, 4-OH & $4-\mathrm{NMe}_{2}$ & 348 & 533 & 0.09 \\
\hline & $3-\mathrm{OH}$ & $\mathrm{H}$ & 336 & 447 & $<0.01$ & & $3-\mathrm{OH}$ & $4-\mathrm{Br}$ & 343 & 451 & $<0.01$ \\
\hline & $3-\mathrm{OH}$ & $2-\mathrm{F}$ & 325 & 445 & $<0.01$ & & $3-\mathrm{OH}$ & $4-\mathrm{Cl}$ & 341 & 447 & $<0.01$ \\
\hline & $3-\mathrm{OH}$ & $3-\mathrm{F}$ & 338 & 443 & $<0.01$ & & $3-\mathrm{OH}$ & 3-OMe & 338 & 442 & $<0.01$ \\
\hline & $3-\mathrm{OH}$ & $4-\mathrm{F}$ & 335 & 444 & $<0.01$ & $3 m$ & $3-\mathrm{OH}$ & 4-OMe & 326 & 420 & $<0.01$ \\
\hline & $3-\mathrm{OH}$ & $3 F, 4-F$ & 341 & 448 & $<0.01$ & & $3-\mathrm{OH}$ & 4- $\mathrm{NMe}_{2}$ & 377 & 542 & 0.05 \\
\hline & $3-\mathrm{OH}$ & $3-\mathrm{Br}$ & 347 & 443 & $<0.01$ & $3 \mathbf{n}^{c}$ & 4-OMe & $2-\mathrm{F}$ & 336 & 419 & 0.71 \\
\hline $3 \mathbf{o}^{c}$ & 4-OMe & 4-OMe & 335 & 409 & 0.77 & $\mathbf{3} \mathbf{q}^{c}$ & 3,4-OMe & 4-OMe & 344 & 448 & 0.74 \\
\hline $\mathbf{3} \mathbf{p}^{c}$ & 4-OMe & 3 -OMe & 306 & 417 & 0.38 & & & & & & \\
\hline
\end{tabular}

${ }^{a}$ Library members without compound numbers represent compounds that were omitted from the text for the sake of brevity. ${ }^{b}$ Data recorded from crude samples. Quantum yields measured in EtOH. External standard: coumarin $120\left(\phi_{\mathrm{r}}=0.88, \lambda_{\mathrm{ex}}=354 \mathrm{~nm}, \lambda_{\mathrm{em}}=435 \mathrm{~nm}\right.$ in EtOH $)$. Error $= \pm 15 \%$. ${ }^{c}$ Synthesized in solution and purified. 


\section{Spectral data for deazalumazines 6.}<smiles>[H][R]1cccc(-c2cc(-c3ccccc3)c3c(=O)n(C)c(=O)n(C)c3n2)c1</smiles>

\begin{tabular}{|c|c|c|c|c|c|c|c|c|c|c|c|}
\hline entry $^{a}$ & $\mathrm{R}^{1}$ & $\mathrm{R}^{2}$ & $\lambda_{\mathrm{ex}}$ & $\lambda_{\mathrm{em}}$ & $\phi_{\mathrm{f}}^{b}$ & entry $^{a}$ & $\mathrm{R}^{1}$ & $\mathrm{R}^{2}$ & $\lambda_{\mathrm{ex}}$ & $\lambda_{\mathrm{em}}$ & $\phi_{\mathrm{f}}^{b}$ \\
\hline $6 \mathbf{a}$ & $4-\mathrm{OH}$ & $\mathrm{H}$ & 362 & 446 & 0.15 & 6d & $4-\mathrm{OH}$ & $4-\mathrm{Br}$ & 364 & 450 & 0.09 \\
\hline \multirow[t]{2}{*}{$6 \mathbf{b}$} & $4-\mathrm{OH}$ & $2-\mathrm{F}$ & 363 & 443 & 0.18 & & $4-\mathrm{OH}$ & $4-\mathrm{Cl}$ & 364 & 449 & 0.10 \\
\hline & $4-\mathrm{OH}$ & $3-\mathrm{F}$ & 364 & 450 & 0.15 & & $4-\mathrm{OH}$ & $3-\mathrm{Cl}$ & 363 & 451 & 0.13 \\
\hline \multirow[t]{3}{*}{$6 c^{c}$} & $4-\mathrm{OH}$ & $4-\mathrm{F}$ & 364 & 445 & 0.19 & & $4-\mathrm{OH}$ & 3-OMe & 363 & 444 & 0.04 \\
\hline & $4-\mathrm{OH}$ & $3 \mathrm{~F}, 4-\mathrm{F}$ & 364 & 450 & 0.12 & $6 e$ & $4-\mathrm{OH}$ & 4-OMe & 365 & 438 & 0.03 \\
\hline & $4-\mathrm{OH}$ & $3-\mathrm{Br}$ & 364 & 452 & 0.07 & & 3-OMe, 4-OH & $3-\mathrm{Br}$ & 370 & 489 & 0.03 \\
\hline \multirow[t]{3}{*}{$6 \mathrm{~g}$} & $3-\mathrm{OMe}, 4-\mathrm{OH}$ & $\mathrm{H}$ & 368 & 480 & 0.06 & $\mathbf{6 j}$ & $3-\mathrm{OMe}, 4-\mathrm{OH}$ & $4-\mathrm{Br}$ & 372 & 495 & 0.05 \\
\hline & 3-OMe, 4-OH & $2-\mathrm{F}$ & 371 & 485 & 0.06 & & 3-OMe, 4-OH & $4-\mathrm{Cl}$ & 371 & 492 & 0.05 \\
\hline & $3-\mathrm{OMe}, 4-\mathrm{OH}$ & $3-\mathrm{F}$ & 370 & 493 & 0.05 & & $3-\mathrm{OMe}, 4-\mathrm{OH}$ & $3-\mathrm{Cl}$ & 372 & 496 & 0.04 \\
\hline \multirow[t]{5}{*}{$6 \mathbf{i}$} & $3-\mathrm{OMe}, 4-\mathrm{OH}$ & $4-\mathrm{F}$ & 370 & 483 & 0.07 & $6 h$ & $3-\mathrm{OMe}, 4-\mathrm{OH}$ & 3-OMe & 370 & 482 & 0.05 \\
\hline & $3-\mathrm{OMe}, 4-\mathrm{OH}$ & $3 \mathrm{~F}, 4-\mathrm{F}$ & 371 & 492 & 0.05 & $6 \mathbf{k}$ & $3-\mathrm{OMe}, 4-\mathrm{OH}$ & 4-OMe & 372 & 474 & 0.06 \\
\hline & $3-\mathrm{OH}$ & $\mathrm{H}$ & 363 & 441 & $<0.01$ & & $3-\mathrm{OH}$ & $4-\mathrm{Br}$ & 355 & 442 & $<0.01$ \\
\hline & $3-\mathrm{OH}$ & $2-\mathrm{F}$ & 365 & 439 & $<0.01$ & $6 s$ & $3-\mathrm{OH}$ & $4-\mathrm{Cl}$ & 356 & 440 & $<0.01$ \\
\hline & $3-\mathrm{OH}$ & $3-\mathrm{F}$ & 354 & 455 & $<0.01$ & & $3-\mathrm{OH}$ & $3-\mathrm{OMe}$ & 347 & 428 & $<0.01$ \\
\hline \multirow[t]{2}{*}{$6 r$} & $3-\mathrm{OH}$ & $4-\mathrm{F}$ & 367 & 441 & 0.05 & & $3-\mathrm{OH}$ & 4-OMe & 351 & 456 & 0.01 \\
\hline & $3-\mathrm{OH}$ & $3 \mathrm{~F}, 4-\mathrm{F}$ & 361 & 446 & $<0.01$ & & $3-\mathrm{OH}$ & $3-\mathrm{Br}$ & 352 & 440 & $<0.01$ \\
\hline $\mathbf{6 t}^{c}$ & 4-OMe & $4-F$ & 360 & 424 & $0.19^{b}$ & & & & & & \\
\hline
\end{tabular}

${ }^{a}$ Library members without compound numbers represent compounds that were omitted from the text for the sake of brevity. ${ }^{b}$ Data recorded from crude samples. Quantum yields measured in EtOH. External standard: coumarin $120\left(\mathrm{f}_{\mathrm{f}}=0.88,1_{\mathrm{ex}}=354 \mathrm{~nm}, 1_{\mathrm{em}}=435 \mathrm{~nm}\right.$ in $\left.\mathrm{EtOH}\right)$. Error $= \pm 15 \%$. ${ }^{c}$ Synthesized in solution and purified. 
Dependence of the spectral properties of cyanopyridines $3 \mathrm{e}, 3 \mathrm{f}, 3 \mathrm{n}$, and $30 \mathrm{on} \mathrm{pH}$.

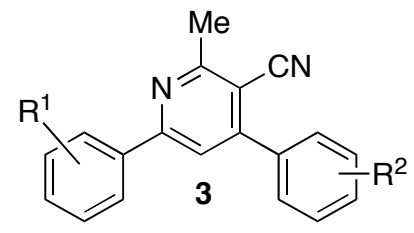

\begin{tabular}{cccccccc}
\hline compound $^{a}$ & $\mathrm{R}^{1}$ & $\mathrm{R}^{2}$ & buffer (in EtOH) & $\mathrm{pH}$ & $\lambda_{\text {ex }}$ & $\lambda_{\text {em }}$ & $\phi_{\mathrm{f}}^{b}$ \\
\hline 3e & $4-\mathrm{OH}$ & $4-\mathrm{OMe}$ & $0.1 \mathrm{M} \mathrm{TFA}$ & 1.6 & 342 & 422 & 0.14 \\
3e & $4-\mathrm{OH}$ & $4-\mathrm{OMe}$ & $0.1 \mathrm{M} \mathrm{AcOH}$ & 4.8 & 339 & 423 & 0.14 \\
3e & $4-\mathrm{OH}$ & $4-\mathrm{OMe}$ & $0.1 \mathrm{M} \mathrm{NH}_{4} \mathrm{OAc}$ & 7.6 & 340 & 423 & 0.12 \\
3e & $4-\mathrm{OH}$ & $4-\mathrm{OMe}$ & $0.1 \mathrm{M} \mathrm{NH}_{4} \mathrm{OAc} / \mathrm{KOH}(1: 1)$ & 10.0 & 340 & 421 & 0.09 \\
\hline 3f & $4-\mathrm{OH}$ & $4-\mathrm{NMe}_{2}$ & $0.1 \mathrm{M} \mathrm{TFA}$ & 1.6 & 336 & 526 & 0.06 \\
3f & $4-\mathrm{OH}$ & $4-\mathrm{NMe}_{2}$ & $0.1 \mathrm{M} \mathrm{AcOH}$ & 4.8 & 336 & 530 & 0.11 \\
3f & $4-\mathrm{OH}$ & $4-\mathrm{NMe}_{2}$ & $0.1 \mathrm{M} \mathrm{NH}_{4} \mathrm{OAc}$ & 7.6 & 335 & 529 & 0.09 \\
3f & $4-\mathrm{OH}$ & $4-\mathrm{NMe}_{2}$ & $0.1 \mathrm{M} \mathrm{NH}_{4} \mathrm{OAc} / \mathrm{KOH}(1: 1)$ & 10.0 & 336 & 528 & 0.09 \\
\hline 3n & $4-\mathrm{OMe}$ & $2-\mathrm{F}$ & $0.1 \mathrm{M} \mathrm{TFA}$ & 1.6 & 337 & 419 & 0.64 \\
3n & $4-\mathrm{OMe}$ & $2-\mathrm{F}$ & $0.1 \mathrm{M} \mathrm{AcOH}^{2}$ & 4.8 & 336 & 419 & 0.65 \\
3n & $4-\mathrm{OMe}$ & $2-\mathrm{F}$ & $0.1 \mathrm{M} \mathrm{NH}_{4} \mathrm{OAc}$ & 7.6 & 336 & 418 & 0.71 \\
3n & $4-\mathrm{OMe}$ & $2-\mathrm{F}$ & $0.1 \mathrm{M} \mathrm{NH}_{4} \mathrm{OAc} / \mathrm{KOH}(1: 1)$ & 10.0 & 336 & 420 & 0.68 \\
\hline 3o & $4-\mathrm{OMe}$ & $4-\mathrm{OMe}$ & $0.1 \mathrm{M} \mathrm{TFA}$ & 1.6 & 335 & 408 & 0.78 \\
3o & $4-\mathrm{OMe}$ & $4-\mathrm{OMe}$ & $0.1 \mathrm{M} \mathrm{AcOH}$ & 4.8 & 337 & 407 & 0.76 \\
3o & $4-\mathrm{OMe}$ & $4-\mathrm{OMe}$ & $0.1 \mathrm{M} \mathrm{NH}_{4} \mathrm{OAc}$ & 7.6 & 337 & 408 & 0.74 \\
30 & $4-\mathrm{OMe}$ & $4-\mathrm{OMe}$ & $0.1 \mathrm{M} \mathrm{NH}_{4} \mathrm{OAc} / \mathrm{KOH}(1: 1)$ & 10.0 & 336 & 408 & 0.76 \\
\hline
\end{tabular}

${ }^{a}$ Data obtained from compounds synthesized in solution and purified. ${ }^{b}$ External standard: coumarin $120\left(\phi_{\mathrm{f}}=0.88, \lambda_{\mathrm{ex}}=354 \mathrm{~nm}, \lambda_{\mathrm{em}}=435 \mathrm{~nm}\right.$ in EtOH $)$. Error $\pm 10 \%$. 


\section{Dependence of the spectral properties of deazalumazines 6c and 6t on pH.}

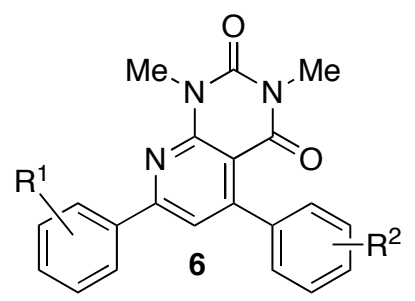

\begin{tabular}{cccccccc}
\hline compound $^{a}$ & $\mathrm{R}^{1}$ & $\mathrm{R}^{2}$ & buffer (in EtOH) & $\mathrm{pH}$ & $\lambda_{\mathrm{ex}}$ & $\lambda_{\mathrm{em}}$ & $\phi_{\mathrm{f}}^{b}$ \\
\hline $\mathbf{6 c}$ & $4-\mathrm{OH}$ & $4-\mathrm{F}$ & $0.1 \mathrm{M} \mathrm{TFA}$ & 1.6 & 362 & 444 & 0.25 \\
$\mathbf{6 c}$ & $4-\mathrm{OH}$ & $4-\mathrm{F}$ & $0.1 \mathrm{M} \mathrm{AcOH}$ & 4.8 & 363 & 444 & 0.25 \\
$\mathbf{6 c}$ & $4-\mathrm{OH}$ & $4-\mathrm{F}$ & $0.1 \mathrm{M} \mathrm{NH} \mathrm{HAC}_{4}$ & 7.6 & 362 & 443 & 0.20 \\
$\mathbf{6 c}$ & $4-\mathrm{OH}$ & $4-\mathrm{F}$ & $0.1 \mathrm{M} \mathrm{NH}_{4} \mathrm{OAc} / \mathrm{KOH}(1: 1)$ & 10.0 & 362 & 442 & 0.16 \\
\hline 6 & $4-\mathrm{OMe}$ & $4-\mathrm{F}$ & $0.1 \mathrm{M} \mathrm{TFA}$ & 1.6 & 359 & 424 & 0.18 \\
$\mathbf{6 t}$ & $4-\mathrm{OMe}$ & $4-\mathrm{F}$ & $0.1 \mathrm{M} \mathrm{AcOH}$ & 4.8 & 360 & 423 & 0.18 \\
$\mathbf{6 t}$ & $4-\mathrm{OMe}$ & $4-\mathrm{F}$ & $0.1 \mathrm{M} \mathrm{NH} 4 \mathrm{OAc}$ & 7.6 & 360 & 425 & 0.20 \\
$\mathbf{6 t}$ & $4-\mathrm{OMe}$ & $4-\mathrm{F}$ & $0.1 \mathrm{M} \mathrm{NH}_{4} \mathrm{OAc} / \mathrm{KOH}(1: 1)$ & 10.0 & 360 & 425 & 0.21 \\
\hline
\end{tabular}

${ }^{a}$ Data obtained from compounds synthesized in solution and purified. ${ }^{b}$ External standard: coumarin $120\left(\phi_{\mathrm{f}}=0.88, \lambda_{\mathrm{ex}}=354 \mathrm{~nm}, \lambda_{\mathrm{em}}=435 \mathrm{~nm}\right.$ in EtOH$)$. Error $\pm 10 \%$. 
Dependence of the spectral properties of dyes 3e, 3f, 3o, 6c, and $6 t$ on solvent.

\begin{tabular}{|c|c|c|c|c|c|c|}
\hline compound $^{a}$ & $\mathrm{R}^{1}$ & $\mathrm{R}^{2}$ & solvent & $\lambda_{\text {ex }}$ & $\lambda_{\mathrm{em}}$ & $\phi_{\mathrm{f}}^{b}$ \\
\hline $3 e$ & $4-\mathrm{OH}$ & 4-OMe & $\mathrm{CHCl}_{3}$ & 333 & 404 & 0.66 \\
\hline $3 e$ & 4-OH & 4-OMe & THF & 339 & 405 & 0.58 \\
\hline $3 e$ & $4-\mathrm{OH}$ & 4-OMe & DMSO & 344 & 440 & 0.31 \\
\hline $3 e$ & $4-\mathrm{OH}$ & 4-OMe & $\mathrm{EtOH}$ & 341 & 424 & 0.15 \\
\hline 3f & 4-OH & $4-\mathrm{NMe}_{2}$ & $\mathrm{CHCl}_{3}$ & 374 & 471 & 0.53 \\
\hline 3f & 4-OH & $4-\mathrm{NMe}_{2}$ & THF & 333 & 486 & 0.53 \\
\hline 3f & 4-OH & $4-\mathrm{NMe}_{2}$ & DMSO & 341 & 538 & 0.17 \\
\hline 3f & 4-OH & 4-NMe ${ }_{2}$ & $\mathrm{EtOH}$ & 334 & 526 & 0.10 \\
\hline 30 & 4-OMe & 4-OMe & $\mathrm{CHCl}_{3}$ & 336 & 393 & 0.74 \\
\hline 30 & 4-OMe & 4-OMe & THF & 337 & 396 & 0.72 \\
\hline 30 & 4-OMe & 4-OMe & DMSO & 340 & 419 & 0.81 \\
\hline 30 & 4-OMe & 4-OMe & EtOH & 335 & 409 & 0.77 \\
\hline $6 c$ & $4-\mathrm{OH}$ & $4-\mathrm{F}$ & $\mathrm{CHCl}_{3}$ & 361 & 417 & 0.13 \\
\hline $6 c$ & 4-OH & $4-\mathrm{F}$ & THF & 361 & 417 & 0.12 \\
\hline $6 c$ & $4-\mathrm{OH}$ & $4-\mathrm{F}$ & DMSO & 367 & 454 & 0.23 \\
\hline $6 c$ & 4-OH & $4-F$ & $\mathrm{EtOH}$ & 363 & 442 & 0.23 \\
\hline $6 \mathrm{t}$ & 4-OMe & $4-F$ & $\mathrm{CHCl}_{3}$ & 361 & 408 & 0.12 \\
\hline $6 t$ & 4-OMe & $4-\mathrm{F}$ & THF & 360 & 410 & 0.09 \\
\hline $6 \mathrm{t}$ & 4-OMe & $4-\mathrm{F}$ & DMSO & 364 & 429 & 0.20 \\
\hline $6 t$ & $4-\mathrm{OMe}$ & $4-\mathrm{F}$ & $\mathrm{EtOH}$ & 361 & 423 & 0.22 \\
\hline
\end{tabular}

${ }^{a}$ Data obtained from compounds synthesized in solution and purified. ${ }^{b}$ External standard: coumarin $120\left(\phi_{\mathrm{f}}=0.88, \lambda_{\mathrm{ex}}=354 \mathrm{~nm}, \lambda_{\mathrm{em}}=435 \mathrm{~nm}\right.$ in EtOH $)$. Error $\pm 10 \%$. 


\section{References and notes.}

(1) Lakowicz, J. R. Principles of Fluorescence Spectroscopy. $2^{\text {nd }}$ ed., New York: Kluwer Academic/Plenum Publishers, 1999.

(2) Still, W. C.; Kahn, M.; Mitra, A. J. Org. Chem. 1978, 43, 2923-2925.

(3) For further information about this microwave reactor system, see: www.milestonesci.com/synth-micro.php

(4) (a) Frank, R. Tetrahedron 1992, 48, 9217-9232. (b) Frank, R. J. Immunol. Methods 2002, 267, 13-26.

(5) Ma, S.; Venanzi, L. M. Tetrahedron Lett. 1993, 34, 5269-5272.

(6) (a) Volkmer-Engert, R.; Hoffmann, B.; Schneider-Mergener, J. Tetrahedron Lett. 1997, 38, 1029-1032. (b) Licha, K.; Bhargava, S.; Rheinlander, C.; Becker, A.; SchneiderMergener, J.; Volkmer-Engert, R. Tetrahedron Lett. 2000, 41, 1711-1715.

(7) Ast, T.; Heine, N.; Germeroth, L.; Schneider-Mergener, J.; Wenschuh, H. Tetrahedron Lett. 1999, 40, 4317-4318.

(8) Carpino, L. A.; Han, G. Y. J. Org. Chem. 1972, 37, 3404-3409.

(9) The decanted linker solution can be poured onto a second sheet of amino cellulose. Immersion overnight in the solution will give a sheet with similar loadings to the primary sheet.

(10) Scharn, D.; Wenschuh, H.; Reineke, U.; Schneider-Mergener, J.; Germeroth, L. J. Comb. Chem. 2000, 2, 361-369.

(11) Besson, T; Coudert, G.; Guillaumet, G. J. Heterocycl. Chem. 1991, 75, 1517-1523. 